This item was submitted to Loughborough's Research Repository by the author.

Items in Figshare are protected by copyright, with all rights reserved, unless otherwise indicated.

\title{
Nonlinear waves in counter-current gas-liquid film flow
}

PLEASE CITE THE PUBLISHED VERSION

http://dx.doi.org/10.1017/S002211201000618X

PUBLISHER

(c) Cambridge University Press

VERSION

VoR (Version of Record)

LICENCE

CC BY-NC-ND 4.0

REPOSITORY RECORD

Tseluiko, Dmitri, and Serafim Kalliadasis. 2019. "Nonlinear Waves in Counter-current Gas-liquid Film Flow". figshare. https://hdl.handle.net/2134/15490. 
This item was submitted to Loughborough's Institutional Repository (https://dspace.lboro.ac.uk/) by the author and is made available under the following Creative Commons Licence conditions.

\section{creative
commons}

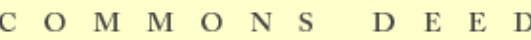

Attribution-NonCommercial-NoDerivs 2.5

You are free:

- to copy, distribute, display, and perform the work

Under the following conditions:

Attribution. You must attribute the work in the manner specified b the author or licensor.

Noncommercial. You may not use this work for commercial purposes.

No Derivative Works. You may not alter, transform, or build upon this work.

- For any reuse or distribution, you must make clear to others the license terms of this work.

- Any of these conditions can be waived if you get permission from the copyright holder.

Your fair use and other rights are in no way affected by the above.

This is a human-readable summary of the Leqal Code (the full license).

\section{Disclaimer 만}

For the full text of this licence, please go to: http://creativecommons.org/licenses/by-nc-nd/2.5/ 


\title{
Nonlinear waves in counter-current gas-liquid film flow
}

\author{
D. TSELUIKO† AND S. KALLIADASIS \\ Department of Chemical Engineering, Imperial College London, London SW7 2AZ, UK
}

(Received 3 June 2010; revised 22 October 2010; accepted 29 November 2010; first published online 18 February 2011)

We investigate the dynamics of a thin laminar liquid film flowing under gravity down the lower wall of an inclined channel when turbulent gas flows above the film. The solution of the full system of equations describing the gas-liquid flow faces serious technical difficulties. However, a number of assumptions allow isolating the gas problem and solving it independently by treating the interface as a solid wall. This permits finding the perturbations to pressure and tangential stresses at the interface imposed by the turbulent gas in closed form. We then analyse the liquid film flow under the influence of these perturbations and derive a hierarchy of model equations describing the dynamics of the interface, i.e. boundary-layer equations, a long-wave model and a weakly nonlinear model, which turns out to be the KuramotoSivashinsky equation with an additional term due to the presence of the turbulent gas. This additional term is dispersive and destabilising (for the counter-current case; stabilizing in the co-current case). We also combine the long-wave approximation with a weighted-residual technique to obtain an integral-boundary-layer approximation that is valid for moderately large values of the Reynolds number. This model is then used for a systematic investigation of the flooding phenomenon observed in various experiments: as the gas flow rate is increased, the initially downward-falling film starts to travel upwards while just before the wave reversal the amplitude of the waves grows rapidly. We confirm the existence of large-amplitude stationary waves by computing periodic travelling waves for the integral-boundary-layer approximation and we corroborate our travelling-wave results by time-dependent computations.

Key words: gas/liquid flow, thin films

\section{Introduction}

Gas-liquid flows are often encountered in a wide variety of natural phenomena and technological applications: from wind-generated waves on the surface of lake and sea waters to heat and mass transfer processes in engineering applications, e.g. evaporators, condensers, heat pipes, cooling towers and chemical reactor columns. In the present study, we consider the problem of a counter-current turbulent gaslaminar liquid flow in a channel with the liquid flowing down one of the channel walls due to gravity. This system has been the subject of active research for more than 60 years, starting from the pioneering experiments of Semyonov (1944). He found

$\dagger$ Present address: School of Mathematics, Loughborough University, Loughborough LE11 3TU, UK.

$\ddagger$ Email address for correspondence: s.kalliadasis@imperial.ac.uk 

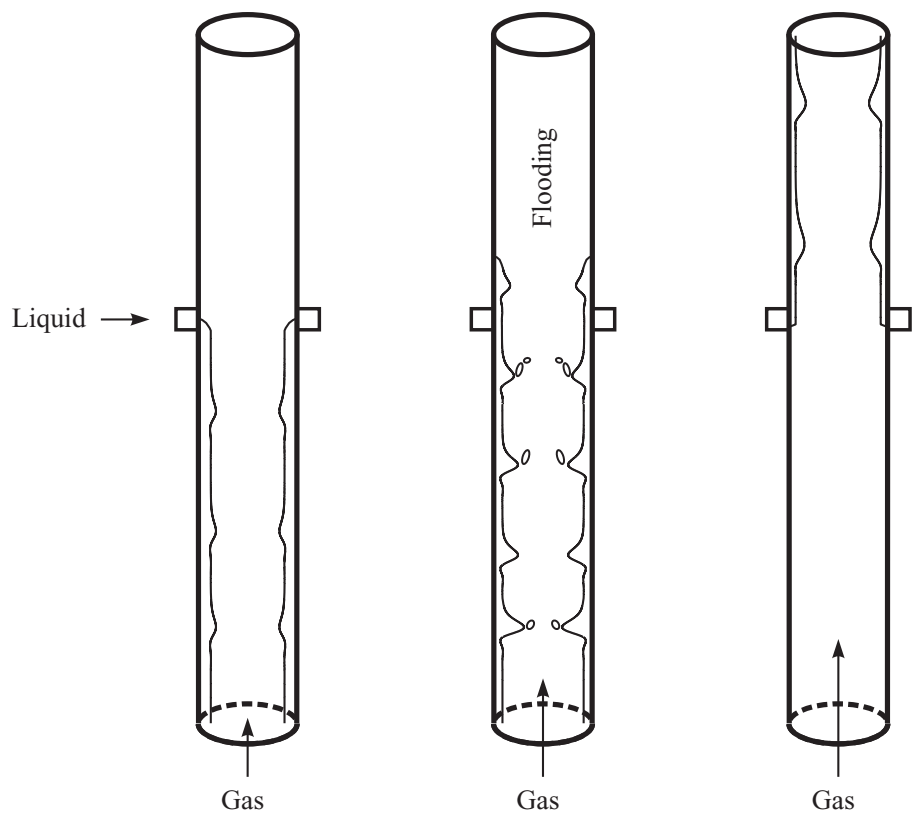

Increasing gas flow rate

FIGURE 1. Schematic representation of the gas-liquid flow in a tube as gas flow rate is increased.

that counter-current turbulent gas-laminar liquid film flow is characterised by a series of unique hydrodynamic phenomena, the most interesting of which is the so-called flooding phenomenon: as the gas flow rate is increased, the initially downward-falling film starts to travel upwards. Also, just before the flow reversal, the amplitude of the interfacial waves grows very rapidly and at the same time the speed of the waves decreases. Eventually, as the flow reverses very rapidly, atomisation of the liquid into the gas phase occurs.

Let us briefly describe the main experimental findings of Semyonov (1944). He considered co-current and counter-current air-water flow in a vertical glass tube of diameter $13.8 \mathrm{~mm}$ and length $2.3 \mathrm{~m}$. The air velocity corresponding to the maximal gas flow rate was $35 \mathrm{~m} \mathrm{~s}^{-1}$, and the typical water film thickness was $50 \mu \mathrm{m}$. A schematic representation is given in figure 1, Semyonov (1944) reported various flow regimes when the air is flowing upwards, the main of which are given below.

(i) Without the air flow, the film flow is laminar with a slightly wavy interface. At slow gas velocities, less than $4 \mathrm{~m} \mathrm{~s}^{-1}$, there are no significant changes in the flow characteristics.

(ii) As the air velocity is increased beyond $4 \mathrm{~m} \mathrm{~s}^{-1}$, the waviness of the interface increases, but the flow is still laminar. Wave rings travel down the pipe separated by $3-5 \mathrm{~cm}$. Sometimes the rings are destroyed as they travel down the pipe. With increasing air flow rate, the velocity of the rings decreases. With increasing liquid flow rate, the velocity of the rings increases. If the air flow rate is slowly increased, then flooding occurs at certain air velocities. The smaller the water flow rate is, the higher air velocity observed before flooding occurs. At a water flow rate of $10^{-2} \mathrm{~kg} \mathrm{~m}^{-1} \mathrm{~s}^{-1}$, the critical air velocity was reported to be $7.3 \mathrm{~m} \mathrm{~s}^{-1}$. 
(iii) If the air velocity is rapidly increased at flooding, the above violent flow characteristics disappear and a thin film starts to flow upwards. The film interface consists of small regular rings travelling upwards. The higher the gas velocity is, the smaller the rings are and the faster they travel.

(iv) If the liquid flow rate is increased, there appear less regular larger rings that travel faster and non-uniformly, and after that the water flow becomes turbulent, a regime beyond the scope of the present study.

Previous work on flooding has been mainly experimental and was devoted to the investigation of the influence of liquid and gas properties as well as channel geometry on the onset of flooding, e.g. Stainthorp (1967), Dukler \& Smith (1979), Lee \& Bankoff (1984), Maron \& Dukler (1984), McQuillan, Whalley \& Hewitt (1985), Larson, Oh \& Chapman (1994), Jayanti, Tokarz \& Hewitt (1996), Sudo (1996), Zapke \& Kröger (2000a,b), Mouza, Pantzali \& Paras (2005), Drosos, Paras \& Karabelas (2006) and Pantzali, Mouza \& Paras (2008), to name but a few. A number of empirical relations which attempt to correlate the gas-liquid flooding transition velocities as a function of the physical and geometrical parameters of the system have also appeared as a result of these experimental studies. It should be noted that although flooding has been the subject of numerous experimental studies over several decades, there still exists controversy in its precise definition and its mechanism. Indeed, it was found that the onset of flooding depends considerably on many factors, such as the channel geometry, channel inclination angle, inlet and outlet conditions and the liquid properties (e.g. Jayanti et al. 1996; Pantzali et al. 2008). We concentrate here on the mechanism associated with the sharp increase of the amplitude of the interfacial waves when the gas velocity gradually approaches the flooding point. The velocity of such waves decreases as the gas velocity increases and the waves can be eventually swept upwards by the gas. This mechanism has been reported in numerous studies, e.g. Semyonov (1944), McQuillan et al. (1985), Jayanti et al. (1996) and Pantzali et al. (2008). Therefore, it is natural to associate the 'onset of flooding' with the formation of almost stationary waves of large amplitude. We adopt this definition of the onset of flooding, and our motivation with the present study is to develop a theoretical framework that would allow a systematic theoretical investigation of this phenomenon.

Previous theoretical works on gas-liquid film flows are limited. Shearer \& Davidson (1965) first stipulated that at the gas velocity corresponding to flooding, a largeamplitude standing wave forms on the liquid surface. To obtain the shape of the standing wave, they used an empirical expression for the variation of the gas pressure. Guguchkin et al. (1979) analysed the linear stability of a liquid film entrained by a turbulent gas that was modelled using the 'quasi-laminarity' assumption of Miles (1957) and Benjamin (1959). Demekhin (1981) treated the problem for the turbulent gas independently of the liquid problem and derived an integral-boundary-layer (IBL) approximation for the liquid, first introduced by Shkadov (1967) for a falling film without a gas. Jurman \& McCready (1989) and Peng, Jurman \& McCready (1991) analysed experimentally and analytically (by performing a linear stability analysis and deriving weakly nonlinear amplitude equations) waves on horizontal thin liquid films sheared by turbulent gas flows.

The approach adopted in the present paper is similar to that of Demekhin (1981) and a recent study by Trifonov (2010), namely the gas problem is solved independently under appropriate assumptions. However, instead of solving the full Navier-Stokes equations for the liquid problem, as in Trifonov (2010), we derive various lowdimensional models, i.e. a long-wave model, a weakly nonlinear model and an IBL approximation based on weighted residual techniques. 
The drawback of the weakly nonlinear model is that it is valid only for smallamplitude waves and sufficiently close to criticality. On the other hand, for the equation resulting from the long-wave expansion, we are close to criticality but the amplitude is not necessarily small. However, for a free-falling film, the timedependent computations of the first-order long-wave evolution equation by Pumir, Manneville \& Pomeau (1983) revealed that the equation exhibits finite-time blow-up behaviour when it is integrated in regions of the parameter space where solitary waves do not exist. This finite-time blow-up was investigated in detail by Oron \& Gottlieb (2002) and Scheid et al. (2004).

Clearly, this behaviour is unphysical and marks the failure of the long-wave evolution equation to correctly describe nonlinear waves far from a close neighbourhood of the critical Reynolds number. A way out is the IBL approximation. For the free-falling film, it was introduced by Shkadov (1967) as noted earlier, following the pioneering theoretical work of Kapitza (1948). However, despite its success in describing nonlinear waves far from criticality, the IBL approximation does have its shortcomings, the principal one being a $20 \%$ error for the critical Reynolds number (except for the vertical plane where the critical Reynolds number vanishes).

The IBL approximation was corrected by Ruyer-Quil \& Manneville (1998, 2000, 2002) who combined the long-wave expansion with a weighted-residual technique using polynomial test functions for the velocity field. It was shown that a simple Galerkin projection with just one test function (the self-similar parabolic profile assumed by Shkadov) and a weight function, the test function itself fully corrects the critical Reynolds number. Subsequently, the weighted-residuals methodology has been extended to falling film problems in the presence of additional effects and complexities, e.g. thermocapillary Marangoni effects induced by heating the substrate either uniformly or non-uniformly (Kalliadasis et al. 2003a; Kalliadasis, Kiyashko \& Demekhin 2003b; Trevelyan \& Kalliadasis 2004b; Ruyer-Quil et al. 2005; Scheid et al. 2005; Trevelyan et al. 2007), solutocapillary Marangoni effects induced by the presence of insoluble surfactants (Pereira \& Kalliadasis 2008), chemical reactions (Trevelyan \& Kalliadasis 2004a) or influence of substrate curvature (Ruyer-Quil et al. 2008).

Compared with the studies by Demekhin (1981) and Trifonov (2010), we use a more accurate model to obtain the perturbations to the shear stress and to the pressure at the interface imposed by the turbulent gas, namely we choose to work in curvilinear boundary-layer coordinates and make use of the quasi-laminarity assumption of Miles (1957) and Benjamin (1959) (Demekhin 1981 and Trifonov 2010 also used the Miles-Benjamin quasi-laminarity assumption but worked in Cartesian coordinates). However, in the works by Miles (1957) and Benjamin (1959), the influence of turbulence comes only through the turbulent mean velocity profile and the usual Navier-Stokes equations are used. This approach is inconsistent in that the mean velocity profile does not satisfy the problem for the unperturbed (flat) interface. Here, we improve the Miles-Benjamin quasi-laminarity approach by using Reynolds-averaged Navier-Stokes equations and curvilinear boundary-layer coordinates. Indeed, analysis in curvilinear coordinates gives significantly better agreement with experiments; see, for example, Thorsness, Morrisroe \& Hanratty (1978) for the comparison of various theoretical approaches for turbulent flow over a wavy wall with experiments. In addition, the model developed by Demekhin (1981) is not self-consistent: it retains the influence of normal pressure of the gas onto the film at the same order with that of tangential stresses, while strictly speaking this should be a higher-order effect as we shall demonstrate here. Finally, our findings based on travelling-wave solutions of the IBL model are supported by time-dependent 


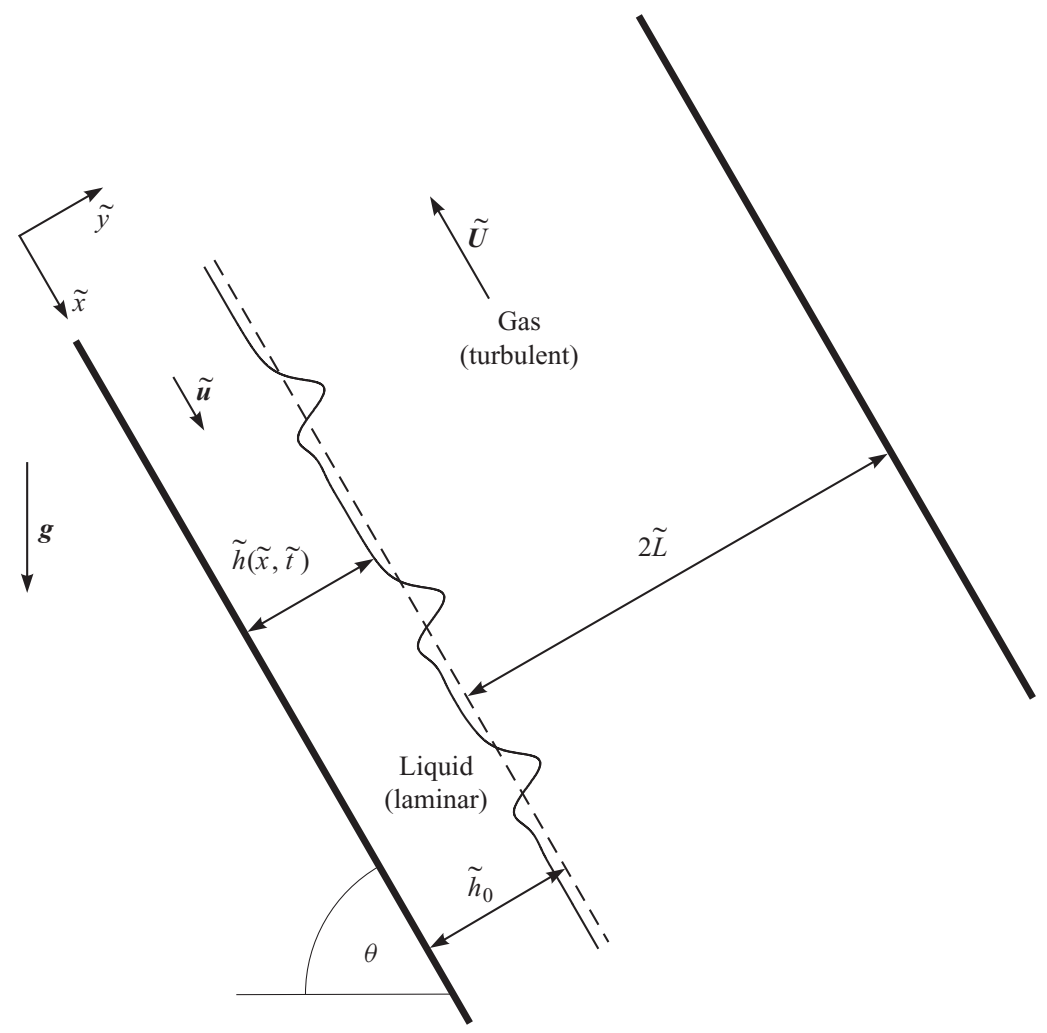

FIGURE 2. Sketch of the profile geometry for the counter-current gas-liquid flow in a channel, where $g=g(\sin \theta,-\cos \theta)$ with $g$ denoting the gravitational acceleration.

computations, unlike Trifonov (2010) who focused on travelling waves only. Moreover, the IBL model has certain advantages over full Navier-Stokes especially for moderate Reynolds numbers: it is a low-dimensional model expressed in terms of interfacial and average quantities which makes it attractive for both mathematical and numerical scrutiny.

This paper is organised as follows. In $\S 2$ we describe the physical setting. In $\S 3$ we analyse the problem for the gas flow. In $\S 4$ we derive various simplified models, including a long-wave model, a weakly nonlinear model and an IBL approximation that combines the long-wave approach with a weighted residuals technique. In $\S 5$ we construct travelling waves of our IBL model and examine their relevance in time-dependent computations. Finally, $\S 6$ is devoted to discussion and conclusions.

\section{Physical setting}

Figure 2 shows the problem definition. We consider a thin liquid film flowing under the action of gravity down the lower wall of an inclined channel forming an angle $\theta$ with the horizontal direction. The liquid has density $\rho_{l}$ and viscosity $\mu_{l}$. A gas of density $\rho_{g}$ and viscosity $\mu_{g}$ flows above the liquid. The liquid film is assumed to be laminar, whilst the gas is taken to be turbulent. Both liquid and gas flows are assumed to be two-dimensional. We introduce a Cartesian coordinate system, $(\tilde{x}, \tilde{y})$, with the $\tilde{x}$-axis pointing along the lower wall of the channel in the direction of the liquid flow. Hereinafter, tildes indicate dimensional variables. The velocity and the 


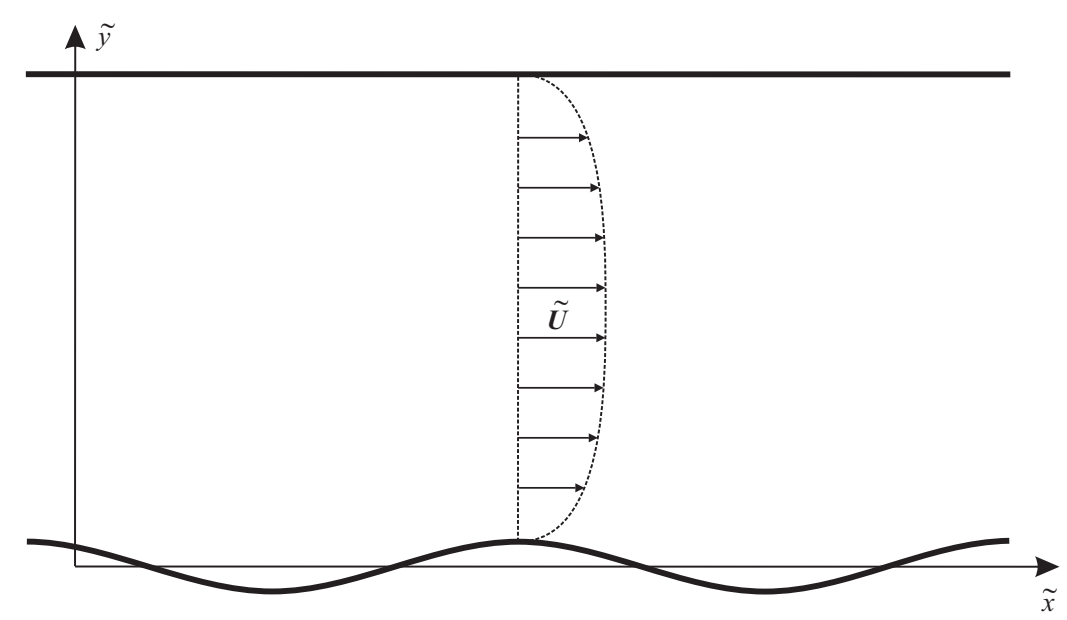

FIGURE 3. Gas flow in a channel with a wavy lower wall.

pressure in the liquid are denoted by $\tilde{\boldsymbol{u}}$ and $\tilde{p}$, respectively, and in the gas by $\widetilde{\boldsymbol{U}}$ and $\widetilde{P}$, respectively. (The precise meaning of the velocity and the pressure in the turbulent gas will be specified below.) The interface is located at $\tilde{y}=\tilde{h}(\tilde{x}, \tilde{t})$, where $\tilde{t}$ denotes time. The undisturbed film thickness is denoted by $\tilde{h}_{0}$ and the distance between the undisturbed interface and the upper wall is denoted by $2 \tilde{L}$.

The solution of the full system of equations governing this gas-liquid flow is an almost formidable problem. However, a number of assumptions and facts allow considerable simplification. First, the dynamic viscosity of the gas, $\mu_{g}$, is much smaller than that of the liquid, $\mu_{l}$. We also assume that the typical velocity in the liquid and of the interfacial waves is much smaller than the typical velocity of the gas. Furthermore, we assume that the turbulent fluctuations in the gas for the gas-liquid flow decay in the same way as the fluctuations in the gas flowing over a rigid wall. These conditions imply that for the gas problem, it is appropriate to model the interface as a solid wall. Therefore, the gas problem can be analysed independently, as discussed in the next section.

\section{Gas problem}

Consider the flow of an incompressible gas in a channel with a flat upper wall and a wavy lower wall (corresponding to the gas-liquid interface as discussed in the previous section). A schematic diagram is given in figure 3 . The gas flows either in the positive or negative $\tilde{x}$-direction. The gas flow is governed by the incompressible Reynolds-averaged Navier-Stokes equations

$$
\rho_{g} \widetilde{\boldsymbol{U}} \cdot \nabla \widetilde{\boldsymbol{U}}=-\nabla \widetilde{\boldsymbol{P}}+\nabla \cdot \widetilde{\boldsymbol{T}}, \quad \nabla \cdot \widetilde{\boldsymbol{U}}=0,
$$

where $\widetilde{\boldsymbol{U}}=(\widetilde{U}, \widetilde{V})$ is the time-averaged gas velocity and $\widetilde{P}$ is the time-averaged pressure. Also, $\widetilde{\boldsymbol{T}}=\tilde{\boldsymbol{\sigma}}+\tilde{\boldsymbol{\tau}}$, where $\tilde{\boldsymbol{\sigma}}=\mu_{g}\left[\nabla \widetilde{\boldsymbol{U}}+(\nabla \widetilde{\boldsymbol{U}})^{\mathrm{T}}\right]$ is the Newtonian stress tensor and $\tilde{\boldsymbol{\tau}}$ is the Reynolds stress tensor due to random turbulent fluctuations in the gas momentum.

The no-slip and no-penetration boundary conditions at the lower and upper walls require

$$
\widetilde{\boldsymbol{U}}=\mathbf{0} \quad \text { at } \quad \tilde{y}=\tilde{h}_{0}+\tilde{s}(\tilde{x}), \quad \tilde{y}=\tilde{h}_{0}+2 \tilde{L},
$$


where $\tilde{s}(\tilde{x})$ represents the disturbance of the gas-liquid interface, which for the gas problem can be considered to be independent of time.

To non-dimensionalise the governing equations, we choose the friction velocity $U^{*}=\sqrt{\left|T_{w}\right| / \rho_{g}}$ as the velocity scale, where $T_{w}$ is the shear stress along the wall for the case when the lower wall is flat, and $\mu_{g} / \rho_{g} U^{*}$ as the length scale. The pressure and the Reynolds stress tensors are scaled by $\rho_{g}\left(U^{*}\right)^{2} \equiv\left|T_{w}\right|$. We drop tildes to denote dimensionless variables and, for simplicity, define the dimensionless coordinate $y$ so that it points along the undisturbed lower wall, i.e. we set $y=\left(\tilde{y}-\tilde{h}_{0}\right) \rho_{g} U^{*} / \mu_{g}$.

The dimensionless governing equations are

$$
\boldsymbol{U} \cdot \boldsymbol{\nabla} \boldsymbol{U}=-\nabla P+\nabla^{2} \boldsymbol{U}+\nabla \cdot \boldsymbol{\tau}, \quad \boldsymbol{\nabla} \cdot \boldsymbol{U}=0 .
$$

In component form, these equations are written as

$$
\begin{aligned}
U U_{x}+V U_{y} & =-P_{x}+U_{x x}+U_{y y}+\tau_{11 x}+\tau_{12 y}, \\
U V_{x}+V V_{y} & =-P_{y}+V_{x x}+V_{y y}+\tau_{12 x}+\tau_{22 y}, \\
U_{x}+V_{y} & =0
\end{aligned}
$$

while the boundary conditions read

$$
U=V=0 \quad \text { at } \quad y=s(x), \quad y=2 L .
$$

\subsection{Base state}

Let us now consider the base state when the lower wall of the channel is flat. In this case, $U=U_{0}(y), V \equiv 0, \tau_{11} \equiv 0, \tau_{22} \equiv 0$ and $\tau_{12}=\tau_{012}(y)$. Because of the midplane symmetry of the channel, we also have $U_{0 y}=0$ and $\tau_{012}=0$ at $y=L$. The governing equations then imply that $P=P_{0}(x)$ and

$$
-P_{0 x}+U_{0 y y}+\tau_{012 y}=0 .
$$

Integrating the latter equation with respect to $y$ from 0 to $L$ yields

$$
-L P_{0 x}-U_{0 y}(0)-\tau_{012}(0)=0,
$$

i.e.

$$
P_{0 x}=-\frac{1}{L}\left[U_{0 y}(0)+\tau_{012}(0)\right] .
$$

The expression in the brackets on the right-hand side of the latter equation is the shear stress at the wall (with a positive or negative sign if the flow is in the positive or negative $x$-direction, respectively). This quantity should be equal to unity due to our choice of non-dimensionalisation. Therefore, we get

$$
P_{0 x}=\mp \frac{1}{L},
$$

where the minus sign corresponds to the case when the gas flows in the positive $x$-direction and the plus sign corresponds to the case when the gas flows in the negative $x$-direction.

By substituting (3.11) into (3.8) and integrating from $y$ to $L$, we obtain the equation

$$
U_{0 y}+\tau_{012}= \pm \frac{L-y}{L},
$$

from which the base velocity profile can be determined, given a particular model for $\tau_{012}$. We will assume that the channel is sufficiently wide and that it is appropriate to 


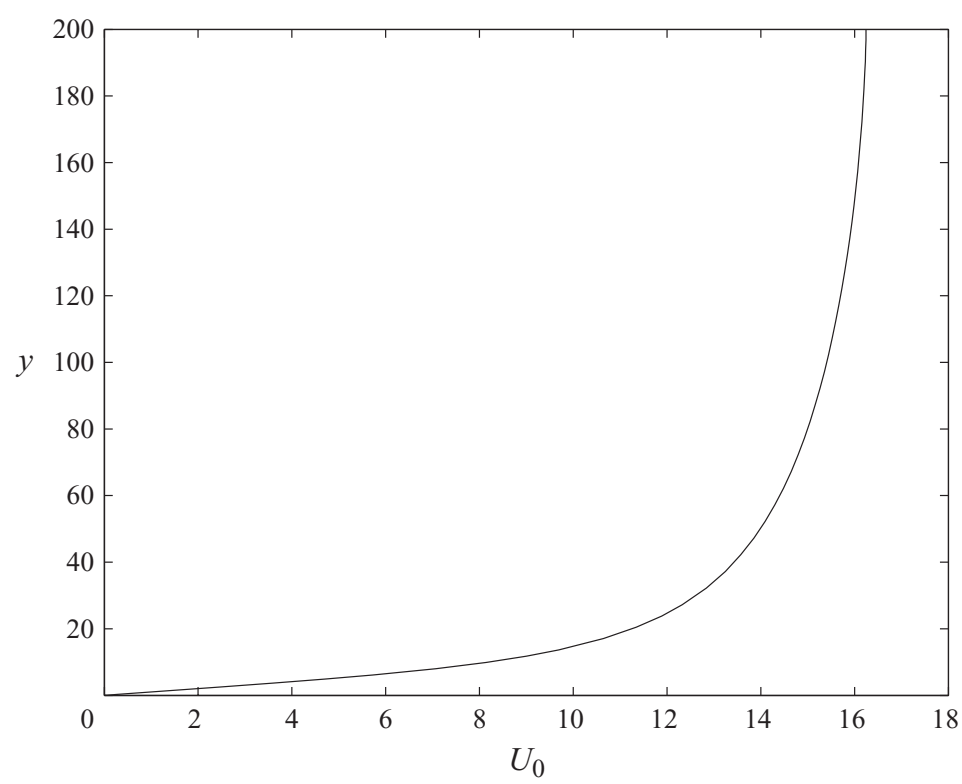

FiguRE 4 . The basic velocity profile for $L=200$.

use the mixing length model for $\tau_{012}$,

$$
\tau_{012}=l^{2}\left|U_{0 y}\right| U_{0 y},
$$

or equivalently,

$$
\tau_{012}=v_{t} U_{0 y},
$$

where $v_{t}=l^{2}\left|U_{0 y}\right|$ is the kinematic eddy viscosity. We then get the following equation:

$$
U_{0 y}+l^{2}\left|U_{0 y}\right| U_{0 y}= \pm \frac{L-y}{L},
$$

where $l$ is the mixing length which can be modelled, for instance, in the wall turbulent boundary layer by the van Driest equation

$$
l=\kappa y[1-\exp (-y / A)],
$$

with $\kappa=0.41$ being the von Kármán constant and $A=25$ (see Schlichting 2000, p. 571). The basic velocity profile for this model is given in figure 4 . The gas flow has a layered structure. It consists of a core layer, where turbulent momentum transfer dominates (the velocity profile is logarithmic in this region), and a thin wall layer where both molecular and turbulent momentum transfer act. There is also a thin viscous sublayer near the wall where turbulent momentum transfer can be neglected (the velocity profile is linear in this region).

\subsection{Flow over a wavy wall of small amplitude}

Next, we will analyse the gas flow for the case when the lower wall is not flat but is wavy of small amplitude, i.e. we assume that $\tilde{s}(\tilde{x}) \equiv \tilde{\epsilon} \mathrm{e}^{\mathrm{i} \tilde{\alpha} \tilde{x}}, \tilde{\epsilon} \ll 1$, where the real part of the latter expression represents the physical wall and $\tilde{\epsilon}$ and $\tilde{\alpha}$ are the amplitude and the wavenumber of the wall undulations, respectively. In dimensionless coordinates, the lower wall is located at $y=s(x) \equiv \epsilon \mathrm{e}^{\mathrm{i} \alpha x}$, where $\epsilon=\tilde{\epsilon} \rho_{g} U^{*} / \mu_{g}$ and $\alpha=\tilde{\alpha} \mu_{g} / \rho_{g} U^{*}$. 


\subsubsection{Benjamin's choice of boundary-layer coordinates}

We choose to work in orthogonal boundary-layer coordinates which, as it turns out, provide significantly better agreement with experiments than the usual Cartesian coordinates. A discussion of general orthogonal curvilinear coordinates is given in the Appendix. We introduce the following coordinate system as in Benjamin (1959):

$$
\begin{aligned}
& \xi_{1}=x_{1}-\mathrm{i} \epsilon \mathrm{e}^{-\alpha x_{2}} \mathrm{e}^{\mathrm{i} \alpha x_{1}}, \\
& \xi_{2}=x_{2}-\epsilon \mathrm{e}^{-\alpha x_{2}} \mathrm{e}^{\mathrm{i} \alpha x_{1}},
\end{aligned}
$$

in which the equation for the lower wall is, to first order in $\epsilon$, given by $\xi_{2}=0$. We note that for simplicity we use complex number notations; however, real parts of the right-hand sides are understood throughout. We also note that by increasing $x_{2}$, the coordinate lines of the curvilinear coordinate system approach exponentially the coordinate lines of the Cartesian system.

We find

$$
\left.\begin{array}{rl}
\left(\begin{array}{ll}
\partial \xi_{1} / \partial x_{1} & \partial \xi_{1} / \partial x_{2} \\
\partial \xi_{2} / \partial x_{1} & \partial \xi_{2} / \partial x_{2}
\end{array}\right) & =\left(\begin{array}{cc}
1+\epsilon \alpha \mathrm{e}^{-\alpha x_{2}} \mathrm{e}^{\mathrm{i} \alpha x_{1}} & \mathrm{i} \epsilon \alpha \mathrm{e}^{-\alpha x_{2}} \mathrm{e}^{\mathrm{i} \alpha x_{1}} \\
-\mathrm{i} \epsilon \alpha \mathrm{e}^{-\alpha x_{2}} \mathrm{e}^{\mathrm{i} \alpha x_{1}} & 1+\epsilon \alpha \mathrm{e}^{-\alpha x_{2}} \mathrm{e}^{\mathrm{i} \alpha x_{1}}
\end{array}\right) \\
& =\left(\begin{array}{cc}
1+\epsilon \alpha \mathrm{e}^{-\alpha \xi_{2}} \mathrm{e}^{\mathrm{i} \alpha \xi_{1}} & \mathrm{i} \epsilon \alpha \mathrm{e}^{-\alpha \xi_{2}} \mathrm{e}^{\mathrm{i} \alpha \xi_{1}} \\
-\mathrm{i} \epsilon \alpha \mathrm{e}^{-\alpha \xi_{2}} \mathrm{e}^{\mathrm{i} \alpha \xi_{1}} & 1+\epsilon \alpha \mathrm{e}^{-\alpha \xi_{2}} \mathrm{e}^{\mathrm{i} \alpha \xi_{1}}
\end{array}\right)+O\left(\epsilon^{2}\right)
\end{array}\right\}
$$

and

$$
\left.\begin{array}{rl}
\left(\begin{array}{ll}
\partial x_{1} / \partial \xi_{1} & \partial x_{1} / \partial \xi_{2} \\
\partial x_{2} / \partial \xi_{1} & \partial x_{2} / \partial \xi_{2}
\end{array}\right) & =\left(\begin{array}{cc}
1+\epsilon \alpha \mathrm{e}^{-\alpha \xi_{2}} \mathrm{e}^{\mathrm{i} \alpha \xi_{1}} & \mathrm{i} \epsilon \alpha \mathrm{e}^{-\alpha \xi_{2}} \mathrm{e}^{\mathrm{i} \alpha \xi_{1}} \\
-\mathrm{i} \epsilon \alpha \mathrm{e}^{-\alpha \xi_{2}} \mathrm{e}^{\mathrm{i} \alpha \xi_{1}} & 1+\epsilon \alpha \mathrm{e}^{-\alpha \xi_{2}} \mathrm{e}^{\mathrm{i} \alpha \xi_{1}}
\end{array}\right)^{-1}+O\left(\epsilon^{2}\right) \\
& =\left(\begin{array}{cc}
1-\epsilon \alpha \mathrm{e}^{-\alpha \xi_{2}} \mathrm{e}^{\mathrm{i} \alpha \xi_{1}} & -\mathrm{i} \epsilon \alpha \mathrm{e}^{-\alpha \xi_{2}} \mathrm{e}^{\mathrm{i} \alpha \xi_{1}} \\
\mathrm{i} \epsilon \alpha \mathrm{e}^{-\alpha \xi_{2}} \mathrm{e}^{\mathrm{i} \alpha \xi_{1}} & 1-\epsilon \alpha \mathrm{e}^{-\alpha \xi_{2}} \mathrm{e}^{\mathrm{i} \alpha \xi_{1}}
\end{array}\right)+O\left(\epsilon^{2}\right) .
\end{array}\right\}
$$

Also, the scale factors are given by

$$
h_{1}=h_{2}=1-\epsilon \alpha \mathrm{e}^{-\alpha \xi_{2}} \mathrm{e}^{\mathrm{i} \alpha \xi_{1}}+O\left(\epsilon^{2}\right),
$$

and the matrix with the entries given by (A 7) is

$$
\left.\begin{array}{rl}
\boldsymbol{\Gamma} & =\left(\begin{array}{cc}
\left(1 / h_{1}\right) \partial x_{1} / \partial \xi_{1} & \left(1 / h_{2}\right) \partial x_{1} / \partial \xi_{2} \\
\left(1 / h_{1}\right) \partial x_{2} / \partial \xi_{1} & \left(1 / h_{2}\right) \partial x_{2} / \partial \xi_{2}
\end{array}\right) \\
& =\left(\begin{array}{cc}
1 & -\mathrm{i} \epsilon \alpha \mathrm{e}^{-\alpha \xi_{2}} \mathrm{e}^{\mathrm{i} \alpha \xi_{1}} \\
\mathrm{i} \epsilon \alpha \mathrm{e}^{-\alpha \xi_{2}} \mathrm{e}^{\mathrm{i} \alpha \xi_{1}} & 1
\end{array}\right)+O\left(\epsilon^{2}\right)
\end{array}\right\}
$$

Using (A 14), we then find

$$
\nabla^{2}=\left(1+2 \epsilon \alpha \mathrm{e}^{-\alpha \xi_{2}} \mathrm{e}^{\mathrm{i} \alpha \xi_{1}}\right)\left[\frac{\partial^{2}}{\partial \xi_{1}^{2}}+\frac{\partial^{2}}{\partial \xi_{2}^{2}}\right]
$$

With the following expansions:

$$
\begin{aligned}
\psi & =\psi_{0}\left(\xi_{2}\right)+\epsilon \psi_{1}\left(\xi_{2}\right) \mathrm{e}^{\mathrm{i} \alpha \xi_{1}}+O\left(\epsilon^{2}\right), \\
P & =P_{0}+\epsilon P_{1}\left(\xi_{2}\right) \mathrm{e}^{\mathrm{i} \alpha \xi_{1}}+O\left(\epsilon^{2}\right), \\
\bar{\tau}_{i j} & =\bar{\tau}_{0 i j}\left(\xi_{2}\right)+\epsilon \bar{\tau}_{1 i j}\left(\xi_{2}\right) \mathrm{e}^{\mathrm{i} \alpha \xi_{1}}+O\left(\epsilon^{2}\right), \quad \text { for } \quad i, j=1,2,
\end{aligned}
$$

where $\psi_{0}$ is the streamfunction corresponding to the base flow chosen so that $\psi_{0}(0)=0$ without loss of generality and bars above the components of $\boldsymbol{\tau}$ indicate that the 
components are taken in curvilinear coordinates, we obtain

$$
\begin{aligned}
& \nabla^{2} \psi=\psi_{0}^{\prime \prime}+\epsilon\left[\psi_{1}^{\prime \prime}-\alpha^{2} \psi_{1}+2 \alpha \mathrm{e}^{-\alpha \xi_{2}} \psi_{0}^{\prime \prime}\right] \mathrm{e}^{\mathrm{i} \alpha \xi_{1}}+O\left(\epsilon^{2}\right), \\
& \nabla^{4} \psi=\psi_{0}^{\prime \prime \prime \prime}+\epsilon\left[\psi_{1}^{\prime \prime \prime \prime}-2 \alpha^{2} \psi_{1}^{\prime \prime}+\alpha^{4} \psi_{1}+4 \alpha \mathrm{e}^{-\alpha \xi_{2}}\left(\psi_{0}^{\prime \prime \prime \prime}-\alpha \psi_{0}^{\prime \prime \prime}\right)\right] \mathrm{e}^{\mathrm{i} \alpha \xi_{1}}+O\left(\epsilon^{2}\right),
\end{aligned}
$$

where primes denote derivatives with respect to $\xi_{2}$. Also, using (A 15) we find

$$
\begin{aligned}
\frac{\partial\left(\psi, \nabla^{2} \psi\right)}{\partial\left(\xi_{1}, \xi_{2}\right)} & =\frac{\partial \psi}{\partial \xi_{1}} \frac{\partial \nabla^{2} \psi}{\partial \xi_{2}}-\frac{\partial \psi}{\partial \xi_{2}} \frac{\partial \nabla^{2} \psi}{\partial \xi_{1}} \\
& =\mathrm{i} \epsilon\left[\alpha \psi_{1} \psi_{0}^{\prime \prime \prime}-\psi_{0}^{\prime}\left(\alpha \psi_{1}^{\prime \prime}-\alpha^{3} \psi_{1}+2 \alpha^{2} \mathrm{e}^{-\alpha \xi_{2}} \psi_{0}^{\prime \prime}\right)\right] \mathrm{e}^{\mathrm{i} \alpha \xi_{2}}+O\left(\epsilon^{2}\right)
\end{aligned}
$$

Next, using $\gamma_{11}=\gamma_{22}=1+O\left(\epsilon^{2}\right), \gamma_{12}=-\mathrm{i} \epsilon \alpha \mathrm{e}^{-\alpha \xi_{2}} \mathrm{e}^{\mathrm{i} \alpha \xi_{1}}+O\left(\epsilon^{2}\right)$ and $\gamma_{21}=\mathrm{i} \epsilon \alpha \mathrm{e}^{-\alpha \xi_{2}} \mathrm{e}^{\mathrm{i} \alpha \xi_{1}}+$ $O\left(\epsilon^{2}\right)$, we obtain

$$
\begin{aligned}
& \tau_{11}=\bar{\tau}_{011}+\epsilon\left[\bar{\tau}_{111}-2 \mathrm{i} \alpha \mathrm{e}^{-\alpha \xi_{2}} \bar{\tau}_{012}\right] \mathrm{e}^{\mathrm{i} \alpha \xi_{1}}+O\left(\epsilon^{2}\right), \\
& \tau_{12}=\bar{\tau}_{012}+\epsilon\left[\bar{\tau}_{112}+\mathrm{i} \alpha \mathrm{e}^{-\alpha \xi_{2}}\left(\bar{\tau}_{011}-\bar{\tau}_{022}\right)\right] \mathrm{e}^{\mathrm{i} \alpha \xi_{1}}+O\left(\epsilon^{2}\right), \\
& \tau_{22}=\bar{\tau}_{022}+\epsilon\left[\bar{\tau}_{122}+2 \mathrm{i} \alpha \mathrm{e}^{-\alpha \xi_{2}} \bar{\tau}_{012}\right] \mathrm{e}^{\mathrm{i} \alpha \xi_{1}}+O\left(\epsilon^{2}\right) .
\end{aligned}
$$

As a result, we find the following expression for $\mathscr{R}$ using (A 19):

$$
\begin{aligned}
\mathscr{R}= & \bar{\tau}_{012}^{\prime \prime}+\epsilon\left[\mathrm{i} \alpha \bar{\tau}_{11}^{\prime}-\mathrm{i} \alpha \bar{\tau}_{122}^{\prime}+\alpha^{2} \bar{\tau}_{112}+\bar{\tau}_{12}^{\prime \prime}+\mathrm{e}^{-\alpha \xi_{2}}\left(2 \mathrm{i} \alpha^{3} \bar{\tau}_{011}-\mathrm{i} \alpha^{2} \bar{\tau}_{011}^{\prime}\right.\right. \\
& \left.\left.-2 \mathrm{i} \alpha^{3} \bar{\tau}_{022}+\mathrm{i} \alpha^{2} \bar{\tau}_{022}^{\prime}-4 \alpha^{3} \bar{\tau}_{012}+2 \alpha^{2} \bar{\tau}_{012}^{\prime}+2 \alpha \bar{\tau}_{012}^{\prime \prime}\right)\right] \mathrm{e}^{\mathrm{i} \alpha \xi_{1}}+O\left(\epsilon^{2}\right) .
\end{aligned}
$$

By substituting (3.21), (3.28), (3.29) and (3.33) into (A 13), we obtain

$$
\begin{aligned}
\psi_{1}^{\prime \prime \prime \prime}-2 \alpha^{2} \psi_{1}^{\prime \prime}+\alpha^{4} \psi_{1}+4 \mathrm{e}^{-\alpha \xi_{2}}\left(\alpha \psi_{0}^{\prime \prime \prime \prime}-\alpha^{2} \psi_{0}^{\prime \prime \prime}\right) \\
=\mathrm{i}\left[\psi_{0}^{\prime}\left(\alpha \psi_{1}^{\prime \prime}-\alpha^{3} \psi_{1}+2 \alpha^{2} \mathrm{e}^{-\alpha \xi_{2}} \psi_{0}^{\prime \prime}\right)-\alpha \psi_{0}^{\prime \prime \prime} \psi_{1}\right]-\mathscr{R}_{1},
\end{aligned}
$$

at first order, where

$$
\begin{aligned}
\mathscr{R}_{1}=\mathrm{i} \alpha \bar{\tau}_{111}^{\prime}-\mathrm{i} \alpha \bar{\tau}_{122}^{\prime}+\alpha^{2} \bar{\tau}_{112}+\bar{\tau}_{12}^{\prime \prime}+\mathrm{e}^{-\alpha \xi_{2}}\left(2 \mathrm{i} \alpha^{3} \bar{\tau}_{011}-\mathrm{i} \alpha^{2} \bar{\tau}_{011}^{\prime}\right. \\
\left.-2 \mathrm{i} \alpha^{3} \bar{\tau}_{022}+\mathrm{i} \alpha^{2} \bar{\tau}_{022}^{\prime}-4 \alpha^{3} \bar{\tau}_{012}+2 \alpha^{2} \bar{\tau}_{012}^{\prime}+2 \alpha \bar{\tau}_{012}^{\prime \prime}\right) .
\end{aligned}
$$

We now adopt a model in which the waviness-induced Reynolds stresses are zero, i.e. $\bar{\tau}_{1 i j}=0, i, j=1,2$, and the normal stresses are neglected, i.e. $\bar{\tau}_{011}=\bar{\tau}_{022}=0$. This assumption was made by Thorsness et al. (1978) in their so-called model A, and it should be noted that the difference with the results obtained in Cartesian coordinates comes exactly from the above assumptions imposed on the components of the Reynolds stress tensor $\boldsymbol{\tau}$ - namely, the above assumptions are not equivalent to assuming that $\tau_{1 i j}=0, i, j=1,2$, and $\tau_{011}=\tau_{022}=0$ in Cartesian coordinates. It is found that the analysis in curvilinear coordinates gives significantly better agreement with experiments compared with that in Cartesian coordinates; see also Thorsness et al. (1978) for the comparison of various theoretical approaches for turbulent flow over a wavy wall with experiments. (However, these authors adopted a different choice of curvilinear coordinates in which the coordinate lines do not approach those of the Cartesian system away from the wall, which seems a rather strange choice.) Our assumption is an improved version of the 'quasi-laminar approximation' of Miles (1957) and Benjamin (1959), who assumed that the only influence of turbulence was through the base velocity profile, i.e. the usual Navier-Stokes equations were used 
and $\mathscr{R}_{1}$ was set to zero (the analysis of Miles 1957 was done in Cartesian coordinates, whilst that of Benjamin 1959 was done in the curvilinear coordinates adopted here), whereas here,

$$
\mathscr{R}_{1}=\mathrm{e}^{-\alpha \xi_{2}}\left(2 \alpha \bar{\tau}_{012}^{\prime \prime}+2 \alpha^{2} \bar{\tau}_{012}^{\prime}-4 \alpha^{3} \bar{\tau}_{012}\right) .
$$

The inconsistency of the above approach by Miles and Benjamin is that the base velocity profile is not the solution of the problem for the unperturbed, i.e. flat, lower wall. We also note that the Miles-Benjamin analysis was done in unbounded domains in the $y$-direction, whilst our analysis is performed for sufficiently wide channels. Finally, we note that our derivation corrects an apparent typo in (3.1) in the work by Benjamin (1959), namely setting $\mathscr{R}_{1} \equiv 0$ and substituting $\psi_{1}\left(\xi_{2}\right)=F\left(\xi_{2}\right)+\mathrm{e}^{-\alpha \xi_{2}} \psi_{0}^{\prime}\left(\xi_{2}\right)$ into (3.34) leads to the same equation for $F$ as (3.1) in Benjamin's work (under replacing $U\left(\xi_{2}\right)-c$ with $\psi_{0}^{\prime}\left(\xi_{2}\right)$ there) with the exception that the term $2 \alpha \psi_{0}^{\prime \prime \prime \prime} \mathrm{e}^{-\alpha \xi_{2}}$, which appears on the right-hand side in the Benjamin equation, is absent in our analysis.

At the wall, the no-slip and no-penetration conditions must be satisfied, which result in the following conditions:

$$
\psi_{1}=0, \quad \psi_{1 \xi_{2}}=0 \quad \text { at } \quad \xi_{2}=0 .
$$

Also, as we move off the wall, the flow must approach the base flow, i.e. for a sufficiently wide channel we can impose the following boundary conditions:

$$
\psi_{1}=0, \quad \psi_{1 \xi_{2}}=0 \quad \text { at } \quad \xi_{2}=L .
$$

Equation (3.34) with the boundary conditions (3.37) and (3.38) can be solved numerically; this was done in Matlab using the boundary-value-problem solver bvp4c. Subsequently, the wall shear stress $\tau_{w}$ and the pressure at the wall $P_{w}$ can be computed. We have

$$
\tau_{w}=\boldsymbol{t} \cdot \boldsymbol{T} \cdot \boldsymbol{n},
$$

where the right-hand side is evaluated at the wall given in curvilinear coordinates by $\xi_{2}=0$ to first order in $\epsilon$. Also, in curvilinear coordinates, $\boldsymbol{t}=(1,0), \boldsymbol{n}=(0,1)$ to first order in $\epsilon$ and $\boldsymbol{T}=\boldsymbol{\sigma}+\boldsymbol{\tau}, \boldsymbol{\tau}=\boldsymbol{0}$ to first order in $\epsilon$. Then, $\tau_{w}=\boldsymbol{t} \cdot \boldsymbol{\sigma} \cdot \boldsymbol{n}=\bar{\sigma}_{12}=\sigma_{12}+O\left(\epsilon^{2}\right)$, and we find

$$
\tau_{w}=\psi_{0}^{\prime \prime}(0)+\epsilon\left[\psi_{1}^{\prime \prime}(0)+\alpha^{2} \psi_{1}(0)+2\left(\alpha \psi_{0}^{\prime \prime}(0)-\alpha^{2} \psi_{0}^{\prime}(0)\right)\right] \mathrm{e}^{\mathrm{i} \alpha \xi_{1}}+O\left(\epsilon^{2}\right) .
$$

Since $\psi_{0}^{\prime}(0)=0, \quad \psi_{0}^{\prime \prime}(0)= \pm 1$, where $+/-$ corresponds to the gas flow in the positive/negative $x$-direction, respectively, and $\psi_{1}(0)=0$, we obtain

$$
\tau_{w}=\tau_{w 0}+\epsilon \tau_{w 1}(\alpha) \mathrm{e}^{\mathrm{i} \alpha \xi_{1}}+O\left(\epsilon^{2}\right),
$$

where

$$
\tau_{w 0}= \pm 1, \quad \tau_{w 1}(\alpha)=\psi_{1}^{\prime \prime}(0) \pm 2 \alpha .
$$

To find the expression for the perturbation of the pressure at the wall, we can use for example,

$$
P_{x_{1}}=-U_{1} U_{1 x_{1}}-U_{2} U_{1 x_{2}}+U_{1 x_{1} x_{1}}+U_{1 x_{2} x_{2}}+\tau_{11 x_{1}}+\tau_{12 x_{2}}
$$

in Cartesian coordinates. Transforming this into curvilinear coordinates, we find at the wall

$$
P_{w 1}(\alpha) \equiv P_{1}(0)=\frac{\mathrm{i}}{\alpha}\left[\alpha P_{0 \xi_{1}}+2 \alpha^{2} \psi_{0}^{\prime \prime}(0)-3 \alpha \psi_{0}^{\prime \prime \prime}(0)-\psi_{1}^{\prime \prime \prime}(0)\right],
$$




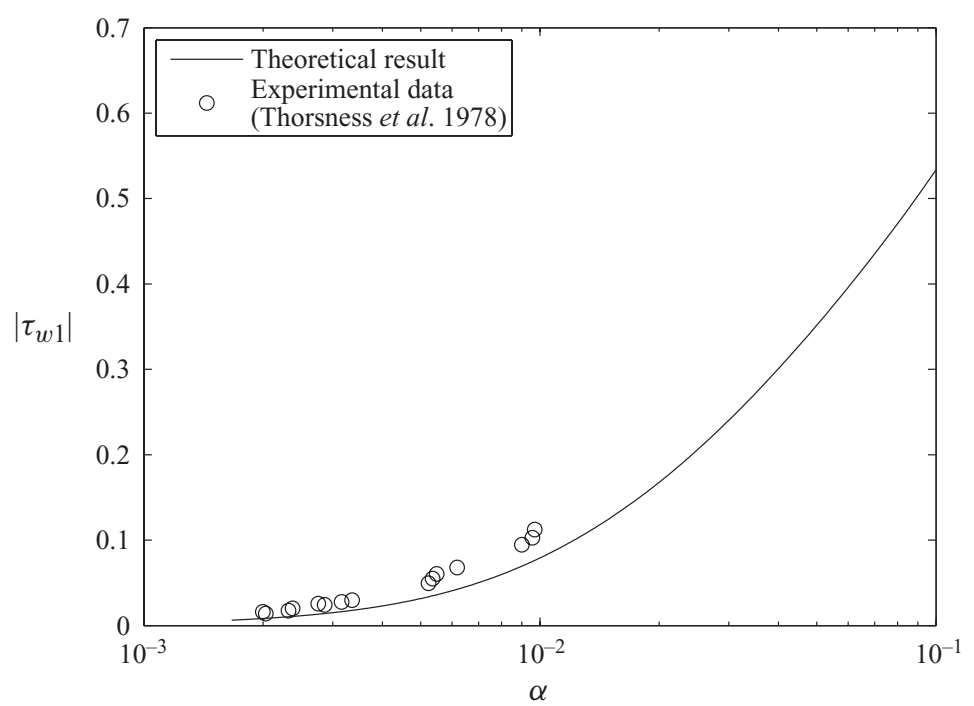

Figure 5. Comparison of our theoretical prediction for $\left|\tau_{w 1}\right|$ with experimental data obtained by Thorsness et al. (1978).

at first order. Using then $P_{0 \xi_{1}}=\mp 1 / L, \psi_{0}^{\prime \prime}(0)= \pm 1$ and $\psi_{0}^{\prime \prime \prime}(0)=\mp 1 / L$, the gas flow in the positive/negative $x$-direction, respectively, we obtain

$$
P_{w 1}(\alpha)=\frac{\mathrm{i}}{\alpha}\left[ \pm \frac{2 \alpha}{L} \pm 2 \alpha^{2}-\psi_{1}^{\prime \prime \prime}(0)\right],
$$

and the pressure at the wall is given by

$$
P_{w}=P_{0}+\epsilon P_{1 w}(\alpha) \mathrm{e}^{\mathrm{i} \alpha \xi_{1}}+O\left(\epsilon^{2}\right)
$$

\subsubsection{Comparison with experiments}

We now compare our theoretical predictions for flow over a wavy wall with experiments. In figures 5 and 6, we compare theoretical predictions for the absolute value and the phase angle in degrees of $\tau_{w 1}$ with experimental results of Thorsness et al. (1978). In figures 7-9, we contrast the tangential stresses at wavy walls found experimentally by Zilker, Cook \& Hanratty (1977) with theoretical predictions. In figures 10 and 11, we compare theoretical predictions for the absolute value and the phase angle in degrees of $P_{w 1}$ with experimental results of various studies (see Thorsness et al. 1978, and references cited therein). In all cases, there is good agreement, even though $\epsilon$ is not small in the experiments of Zilker et al. (1977), suggesting that the simple mixing length model we adopted is capable of capturing satisfactorily turbulent gas flow over a wavy boundary (in the region of moderate Reynolds numbers; $\sim 150-500$ in the experiments). We also note that in the experiments the phase angle of $\tau_{w 1}$ decreases monotonically with $\alpha$, whilst in the theory $\tau_{w 1}$ first increases with $\alpha$ and then decreases monotonically, see figure 6. (Such a trend was also found by Thorsness et al. 1978, for their model A.) Nevertheless, the agreement is still good for not too small values of $\alpha$.

For a periodic wall of an arbitrary shape of zero mean and small amplitude, we presume that the perturbations to the wall shear stress and the pressure depend linearly on the wall shape. This 'linear response' assumption for the gas problem implies that for an arbitrary small-amplitude periodic wall of period $\lambda$ and zero mean 


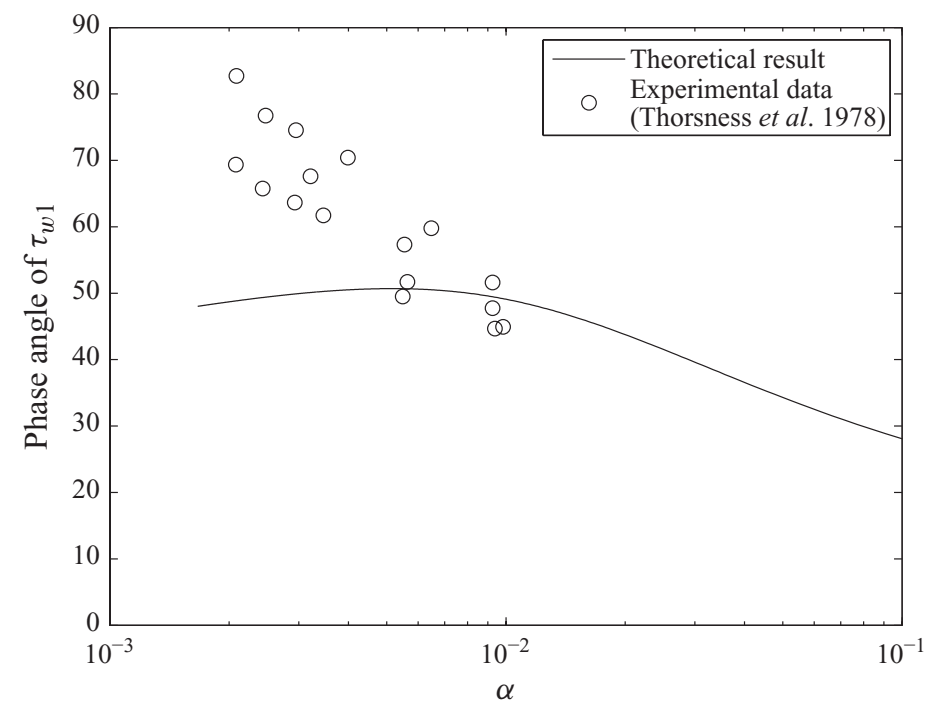

FIGURE 6. Comparison of our theoretical prediction for the phase angle of $\tau_{w 1}$ in degrees with experimental data obtained by Thorsness et al. (1978).

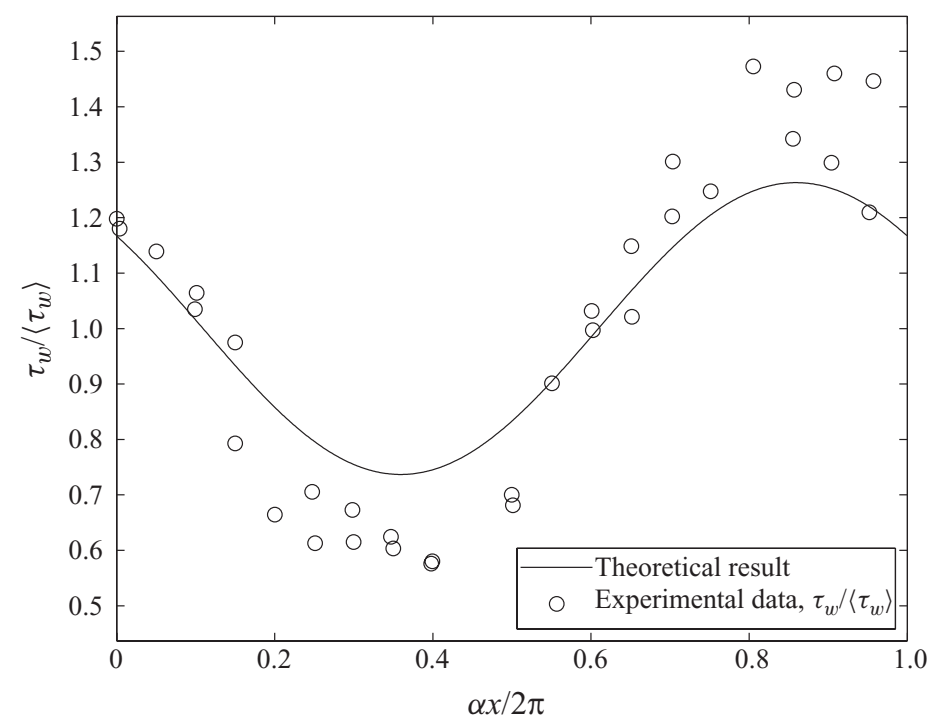

FIGURE 7. Tangential stress distribution at a wavy wall with $\epsilon=6.7, \alpha=0.00584$ and with the gas flowing in the positive $x$-direction. The solid line is the theoretical result $1+\epsilon \operatorname{Re}\left(\tau_{w 1}(\alpha) \mathrm{e}^{\mathrm{i} \alpha x}\right)$ and the circles show $\tau_{w} /\left\langle\tau_{w}\right\rangle$ found experimentally by Zilker et al. (1977), figure 5. Here $\left\langle\tau_{w}\right\rangle$ denotes the average value of the tangential stress at the wall.

written as a superposition of Fourier harmonics,

$$
y=s(x) \equiv \sum_{\substack{n=-\infty \\ n \neq 0}}^{\infty} s_{n} \mathrm{e}^{\mathrm{i} \alpha_{n} x},
$$




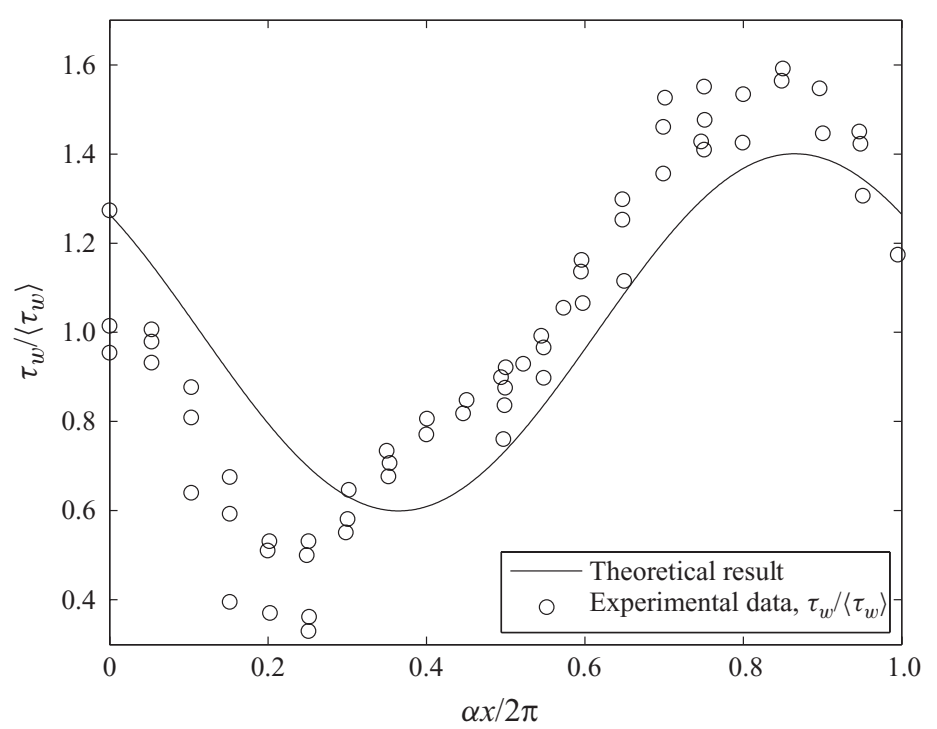

Figure 8. The same results as in figure 7 but with $\epsilon=50.5$ and $\alpha=0.00195$.

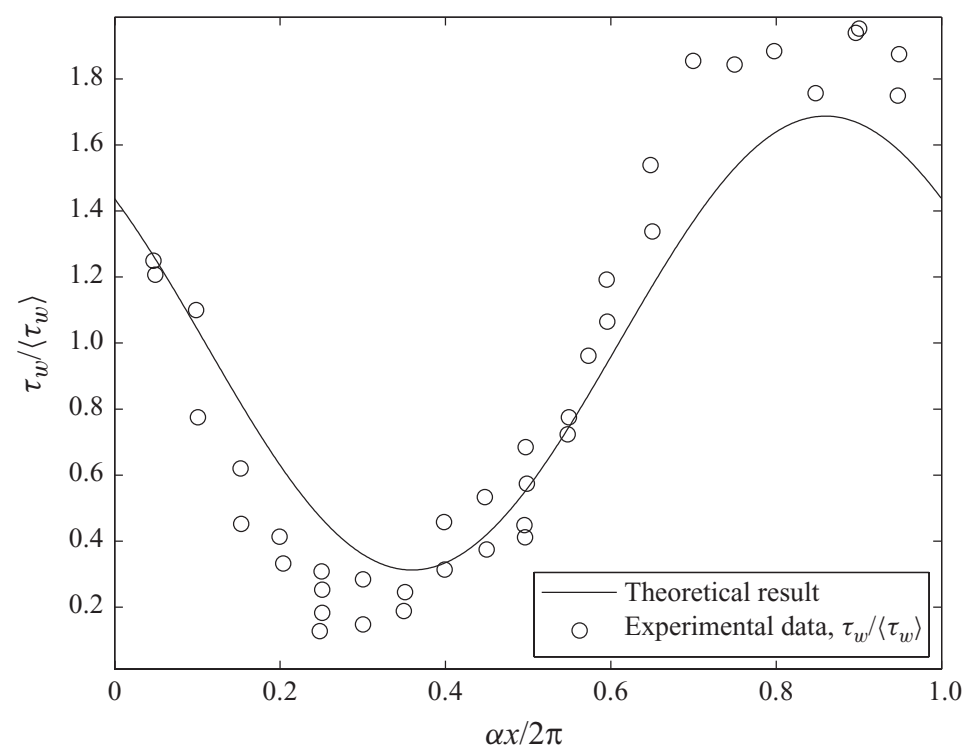

Figure 9. The same results as in figure 7 but with $\epsilon=14.9$ and $\alpha=0.00656$.

where $\alpha_{n}=2 \pi n / \lambda$, the wall shear stress and pressure are also superpositions of Fourier harmonics with the same wavenumbers $\alpha_{n}$ :

$$
\begin{gathered}
\tau_{w}[s]=\tau_{w 0}+\sum_{\substack{n=-\infty \\
n \neq 0}}^{\infty} s_{n} \tau_{w 1}\left(\alpha_{n}\right) \mathrm{e}^{\mathrm{i} \alpha_{n} x}, \\
P_{w}[s]=P_{0}+\sum_{\substack{n=-\infty \\
n \neq 0}}^{\infty} s_{n} P_{w 1}\left(\alpha_{n}\right) \mathrm{e}^{\mathrm{i} \alpha_{n} x} .
\end{gathered}
$$




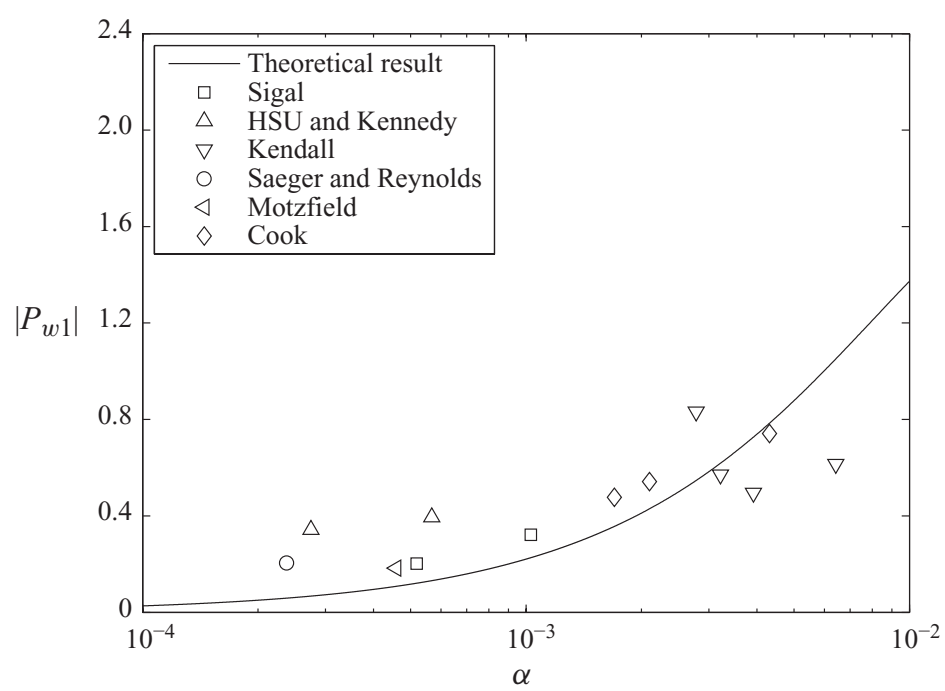

FIGURE 10. Comparison of our theoretical prediction for $\left|P_{w 1}\right|$ with experimental data (see Thorsness et al. 1978).

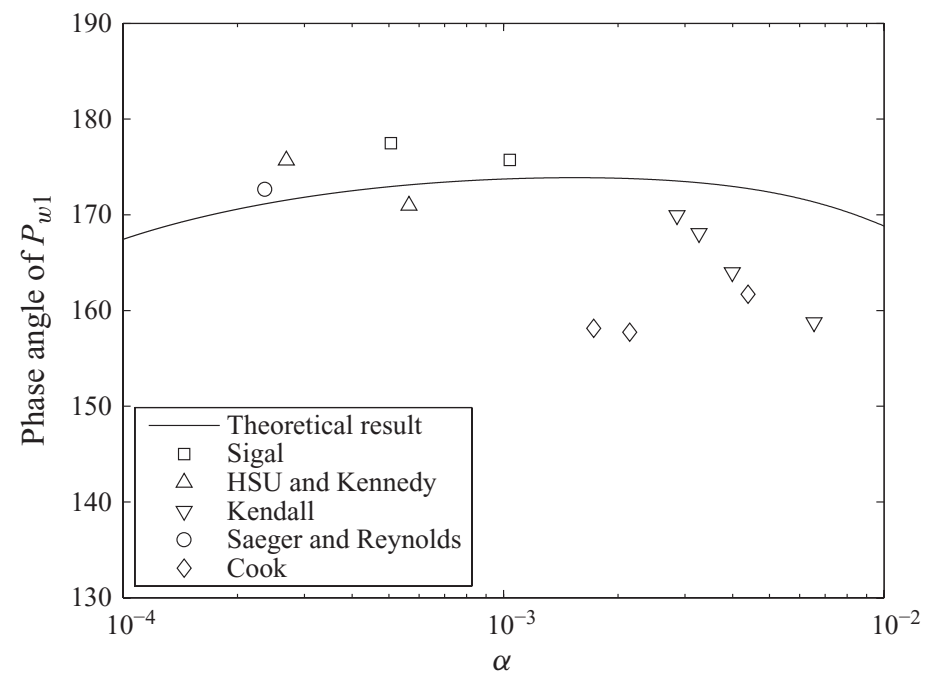

Figure 11. Comparison of our theoretical prediction for the phase angle of $P_{w 1}$ with experimental data (see Thorsness et al. 1978).

It is now useful to convert (3.48) and (3.49) into dimensional forms. For a wall given by

$$
\tilde{y}=\tilde{s}(\tilde{x}) \equiv \sum_{\substack{n=-\infty \\ n \neq 0}}^{\infty} \tilde{s}_{n} \mathrm{e}^{\mathrm{i} \tilde{\alpha}_{n} \tilde{x}}
$$


where $\tilde{s}_{n}=s_{n} \mu_{g} / \rho_{g} U^{*}, \quad \tilde{\alpha}_{n}=\alpha_{n} \rho_{g} U^{*} / \mu_{g}$, we obtain the following dimensional expressions for the wall shear stress and pressure:

$$
\begin{aligned}
& \tilde{\tau}_{w}[\tilde{s}]=\left|T_{w}\right| \tau_{w 0}+\frac{\rho_{g} U^{*}\left|T_{w}\right|}{\mu_{g}} \sum_{\substack{n=-\infty \\
n \neq 0}}^{\infty} \tilde{s}_{n} \tau_{w 1}\left(\tilde{\alpha}_{n} \mu_{g} / \rho_{g} U^{*}\right) \mathrm{e}^{\mathrm{i} \tilde{\alpha}_{n} \tilde{x}}, \\
& \widetilde{P}_{w}[\tilde{s}]=\left|T_{w}\right| P_{0}+\frac{\rho_{g} U^{*}\left|T_{w}\right|}{\mu_{g}} \sum_{\substack{n=-\infty \\
n \neq 0}}^{\infty} \tilde{s}_{n} P_{w 1}\left(\tilde{\alpha}_{n} \mu_{g} / \rho_{g} U^{*}\right) \mathrm{e}^{\mathrm{i} \tilde{\alpha}_{n} \tilde{x}} .
\end{aligned}
$$

\section{Liquid problem}

Having found the shear stress and the pressure at the gas-liquid interface introduced by the turbulent gas flow, we can proceed to the problem for the liquid film. The governing equations are the incompressible Navier-Stokes equations:

$$
\rho_{l}\left(\tilde{\boldsymbol{u}}_{\tilde{t}}+\tilde{\boldsymbol{u}} \cdot \nabla \tilde{\boldsymbol{u}}\right)=-\nabla \tilde{p}+\mu_{l} \nabla^{2} \tilde{\boldsymbol{u}}+\rho_{l} \boldsymbol{g}, \quad \nabla \cdot \tilde{\boldsymbol{u}}=0 .
$$

We recall that $\tilde{\boldsymbol{u}}=(\tilde{u}, \tilde{v})$ is the liquid velocity, $\tilde{p}$ is the pressure, $\rho_{l}$ and $\mu_{l}$ are the density and the viscosity of the liquid, respectively, and $g=(g \sin \theta,-g \cos \theta)$. The no-slip and no-penetration conditions at the wall require that

$$
\tilde{\boldsymbol{u}}=\mathbf{0} \quad \text { at } \quad \tilde{y}=0 .
$$

Kinematic compatibility at the film surface requires that

$$
\tilde{v}=\tilde{h}_{\tilde{t}}+\tilde{u} \tilde{h}_{\tilde{x}}, \quad \text { at } \quad \tilde{y}=\tilde{h}(\tilde{x}, \tilde{t}) .
$$

The tangential stress balance at the film surface is

$$
\boldsymbol{t} \cdot \tilde{\boldsymbol{\sigma}}_{1} \cdot \boldsymbol{n}=\tilde{\tau}_{w}[\tilde{h}], \quad \text { at } \quad \tilde{y}=\tilde{h}(\tilde{x}, \tilde{t}),
$$

where $\boldsymbol{n}$ and $\boldsymbol{t}$ are unit vectors, normal (pointing into the gas) and tangent to the interface, respectively, $\tilde{\boldsymbol{\sigma}}_{1}=\mu_{l}\left(\nabla \tilde{\boldsymbol{u}}+\nabla \tilde{\boldsymbol{u}}^{\mathrm{T}}\right)$ is the Newtonian stress tensor in the liquid and $\tilde{\tau}_{w}[\tilde{h}]$ is the tangential stress exerted onto the interface by the turbulent gas, which was found in the previous section. Finally, the normal stress balance demands

$$
\tilde{p}=\widetilde{P}_{w}[\tilde{h}]+\gamma \kappa+\boldsymbol{n} \cdot \tilde{\boldsymbol{\sigma}}_{1} \cdot \boldsymbol{n}, \quad \text { at } \quad \tilde{y}=\tilde{h}(\tilde{x}, \tilde{t}),
$$

where $\gamma$ is the surface tension of the liquid, $\kappa$ is the curvature of the interface taken to be positive when the surface is concave downwards and $\widetilde{P}_{w}[\tilde{h}]$ is the pressure exerted onto the interface by the turbulent gas, which was found in the previous section.

The base solution corresponding to a flat interface, i.e. $\tilde{h}(\tilde{x}, \tilde{t}) \equiv \tilde{h}_{0}$, is given by

$$
\begin{gathered}
\tilde{u}=\frac{1}{2}\left(\frac{\left|T_{w}\right| P_{0 \tilde{x}}}{\mu_{l}}-\frac{\rho_{l} \mathrm{~g} \sin \theta}{\mu_{l}}\right)\left(\tilde{y}-2 \tilde{h}_{0}\right) \tilde{y}+\frac{\left|T_{w}\right| \tau_{w 0}}{\mu_{l}} \tilde{y}, \quad \tilde{v}=0, \\
\tilde{p}=\left|T_{w}\right| P_{0}-\rho_{l} \mathrm{~g} \cos \theta\left(\tilde{y}-\tilde{h}_{0}\right) .
\end{gathered}
$$

To non-dimensionalise the liquid problem, we choose $\tilde{h}_{0}$ as the length scale, the Nusselt surface speed of a flat free-falling film in the absence of the gas, $\tilde{u}_{0}=\rho_{l} \mathrm{~g} \tilde{h}_{0}^{2} \sin \theta / 2 \mu_{l}$, as the velocity scale, $\tilde{h}_{0} / \tilde{u}_{0}$ as the time scale and $\mu_{l} \tilde{u}_{0} / \tilde{h}_{0}$ as the pressure scale. Again, we drop tildes to denote dimensionless variables (it should be noted that the dimensionless variables used in this section should not be confused 
with those used in the previous section). The dimensionless Navier-Stokes equations then written in component form are

$$
\begin{gathered}
\operatorname{Re}\left(u_{t}+u u_{x}+v u_{y}\right)=-p_{x}+u_{x x}+u_{y y}+2, \\
\operatorname{Re}\left(v_{t}+u v_{x}+v v_{y}\right)=-p_{y}+v_{x x}+v_{y y}-2 \cot \theta, \\
u_{x}+v_{y}=0 .
\end{gathered}
$$

At the wall we have

$$
u=v=0 \quad \text { at } \quad y=0,
$$

and at the interface we have

$$
\begin{gathered}
v=h_{t}+u h_{x}, \quad \text { at } \quad y=h, \\
-\frac{1}{1+h_{x}^{2}}\left[2\left(u_{x}-v_{y}\right) h_{x}-\left(1-h_{x}^{2}\right)\left(u_{y}+v_{x}\right)\right]=\hat{\tau}_{w}[h], \quad \text { at } \quad y=h, \\
p=\widehat{P}_{w}[h]-\frac{W e R e h_{x x}}{\left(1+h_{x}^{2}\right)^{3 / 2}}+\frac{2}{1+h_{x}^{2}}\left[u_{x} h_{x}^{2}-h_{x}\left(u_{y}+v_{x}\right)+v_{y}\right], \quad \text { at } \quad y=h,
\end{gathered}
$$

where the Reynolds number, $R e$, and the Weber number, We, are defined respectively as

$$
R e=\frac{\rho_{l} \tilde{u}_{0} \tilde{h}_{0}}{\mu_{l}}, \quad W e=\frac{\gamma}{\rho_{l} \tilde{u}_{0}^{2} \tilde{h}_{0}} .
$$

For convenience, we write

$$
R e=\frac{R e_{0} \sin \theta}{2}, \quad W e=\frac{4 K a}{R e_{0}^{5 / 3} \sin ^{2} \theta},
$$

where

$$
R e_{0}=\frac{\rho_{l}^{2} \mathrm{~g} \tilde{h}_{0}^{3}}{\mu_{l}^{2}}, \quad K a=\frac{\gamma \rho_{l}^{1 / 3}}{\mathrm{~g}^{1 / 3} \mu_{l}^{4 / 3}}
$$

are the modified Reynolds number and the Kapitza number, respectively (Pereira \& Kalliadasis 2008). It is only the modified Reynolds number that depends on $\tilde{h}_{0}$ which is a flow control parameter; the Kapitza number depends only on the physical properties of the liquid.

For a periodic interface given by

$$
h(x, t)=1+\sum_{\substack{n=-\infty \\ n \neq 0}}^{\infty} h_{n}(t) \mathrm{e}^{\mathrm{i} \beta_{n} x}
$$

where $\beta_{n}=2 \pi n / \lambda$, with $\lambda=\tilde{\lambda} / \tilde{h}_{0}$ denoting the dimensionless period, we have

$$
\hat{\tau}_{w}[h]=\frac{\tilde{h}_{0}}{\mu_{l} \tilde{u}_{0}} \tilde{\tau}_{w}[\tilde{h}]=\hat{\tau}_{w 0}+\left|\hat{\tau}_{w 0}\right| \nu \hat{\tau}_{w 1}[h],
$$

where

$$
\hat{\tau}_{w 0}=\frac{\tilde{h}_{0}\left|T_{w}\right| \tau_{w 0}}{\mu_{l} \tilde{u}_{0}}, \quad v=\frac{\tilde{h}_{0} \rho_{g} U^{*}}{\mu_{g}}, \quad \hat{\tau}_{w 1}[h] \equiv \sum_{\substack{n=-\infty \\ n \neq 0}}^{\infty} h_{n} \tau_{w 1}\left(\beta_{n} / v\right) \mathrm{e}^{\mathrm{i} \beta_{n} x} .
$$


Note that $v$ is the ratio of the length scales used to non-dimensionalise the liquid and the gas problems, respectively. Also,

$$
\widehat{P}_{w}[h]=\frac{h_{0}}{\mu_{l} \tilde{u}_{0}} \widetilde{P}_{w}[\tilde{h}]=\widehat{P}_{0}+\left|\hat{\tau}_{w 0}\right| \nu \widehat{P}_{1}[h],
$$

where

$$
\widehat{P}_{0}=\left|\hat{\tau}_{w 0}\right| P_{0}=\widehat{P}_{0}^{a}+\widehat{P}_{0}^{b} x, \quad \widehat{P}_{1}[h] \equiv \sum_{\substack{n=-\infty \\ n \neq 0}}^{\infty} h_{n} P_{w 1}\left(\beta_{n} / v\right) \mathrm{e}^{\mathrm{i} \beta_{n} x} .
$$

Recall that

$$
P_{0}=P_{0}^{a} \mp \frac{1}{\hat{L}} x,
$$

where $P_{0}^{a}$ is a constant reference pressure, $\hat{L}=\tilde{L} / \tilde{h}_{0}$ is the dimensionless channel width and the minus/plus sign corresponds to the positive/negative gas direction, respectively. Hence,

$$
\widehat{P}_{0}^{a}=\left|\hat{\tau}_{w 0}\right| P_{0}^{a}, \quad \widehat{P}_{0}^{b}=-\frac{\hat{\tau}_{w 0}}{\hat{L}} .
$$

The dimensionless base solution then is

$$
\begin{gathered}
u=\left(-\frac{\widehat{P}_{0}^{b}}{2}-1\right)(y-2) y+\hat{\tau}_{w 0} y, \quad v=0, \\
p=\widehat{P}_{0}-2 \cot \theta(y-1) .
\end{gathered}
$$

Furthermore, we can write

$$
\hat{\tau}_{w 0}=\frac{2}{R e_{0}^{1 / 3} \sin \theta} \Theta \tau_{w 0}, \quad v=N \operatorname{Re}_{0}^{1 / 3} \Theta^{1 / 2},
$$

where

$$
\Theta=\frac{\left|T_{w}\right|}{\rho_{l}^{1 / 3} \mathrm{~g}^{2 / 3} \mu_{l}^{2 / 3}}, \quad N=\frac{\mu_{l} \rho_{g}^{1 / 2}}{\mu_{g} \rho_{l}^{1 / 2}} .
$$

The parameter $\Theta$ controls the gas shear-stress strength, whilst the parameter $N$ depends only on the densities and viscosities of the liquid and the gas.

Let us consider, for example, helium as a gas and methanol as a liquid at room temperature. These fluids, amongst others, were used in the experiments of Zapke \& Kröger $(2000 a, b)$, who investigated the effect of the fluid properties and the channel geometry on flooding in rectangular ducts. At room temperature, we have

$$
\begin{gathered}
\rho_{g}=1.65 \times 10^{-1} \mathrm{~kg} \mathrm{~m}^{-3}, \quad \mu_{g}=2 \times 10^{-5} \mathrm{~Pa} \mathrm{~s}, \\
\rho_{l}=791 \mathrm{~kg} \mathrm{~m}^{-3}, \quad \mu_{l}=0.575 \times 10^{-3} \mathrm{~Pa} \mathrm{~s}, \quad \gamma=22 \times 10^{-3} \mathrm{~N} \mathrm{~m}^{-1},
\end{gathered}
$$

which gives

$$
K a \approx 1988.5, \quad N \approx 0.4
$$

i.e. we have

$$
W e=\frac{7954}{R e_{0}^{5 / 3} \sin ^{2} \theta}, \quad v=0.4 R e_{0}^{1 / 3} \Theta^{1 / 2} .
$$

Next, assuming long waves, we write

$$
x=\frac{\xi}{\epsilon}, \quad t=\frac{\tau}{\epsilon}, \quad v=\epsilon w,
$$


where $\epsilon \ll 1$ is the so-called long-wave or film parameter, which is usually defined as the ratio of the undisturbed film thickness to the length scale over which variations in the streamwise direction occur. This so-called long-wave approximation has been central to thin-film studies (see e.g. Oron, Davis \& Bankoff 1997; Kalliadasis \& Thiele 2007; Craster \& Matar 2009). We then obtain

$$
\begin{gathered}
\epsilon \operatorname{Re}\left(u_{\tau}+u u_{\xi}+w u_{y}\right)=-\epsilon p_{\xi}+\epsilon^{2} u_{\xi \xi}+u_{y y}+2, \\
\epsilon^{2} \operatorname{Re}\left(w_{\tau}+u w_{\xi}+w w_{y}\right)=-p_{y}+\epsilon^{3} w_{\xi \xi}+\epsilon w_{y y}-2 \cot \theta, \\
u_{\xi}+w_{y}=0,
\end{gathered}
$$

subject to

and

$$
u=w=0 \quad \text { at } \quad y=0,
$$

$$
\begin{gathered}
w=h_{\tau}+u h_{\xi} \quad \text { at } \quad y=h \\
-\frac{1}{1+\epsilon^{2} h_{\xi}^{2}}\left[2 \epsilon^{2}\left(u_{\xi}-w_{y}\right) h_{\xi}-\left(1-\epsilon^{2} h_{\xi}^{2}\right)\left(u_{y}+\epsilon^{2} w_{\xi}\right)\right]=\hat{\tau}_{w}[h] \quad \text { at } \quad y=h \\
\left.p=\widehat{P}_{w}[h]-\frac{\epsilon^{2} W e R e h_{\xi \xi}}{\left(1+\epsilon^{2} h_{\xi}^{2}\right)^{3 / 2}}+\frac{2}{1+\epsilon^{2} h_{\xi}^{2}}\left[\epsilon^{3} u_{\xi} h_{\xi}^{2}-\epsilon h_{\xi}\left(u_{y}+\epsilon^{2} w_{\xi}\right)+\epsilon w_{y}\right] \quad \text { at } \quad y=h .39\right)
\end{gathered}
$$

We also note that

$$
\widehat{P}_{1}[h] \equiv \sum_{\substack{n=-\infty \\ n \neq 0}}^{\infty} h_{n} P_{w 1}\left(\frac{\bar{\beta}_{n}}{\nu / \epsilon}\right) \mathrm{e}^{\mathrm{i} \bar{\beta}_{n} \xi}, \quad \hat{\tau}_{w 1}[h] \equiv \sum_{\substack{n=-\infty \\ n \neq 0}}^{\infty} h_{n} \tau_{w 1}\left(\frac{\bar{\beta}_{n}}{\nu / \epsilon}\right) \mathrm{e}^{\mathrm{i} \bar{\beta}_{n} \xi},
$$

where $\bar{\beta}_{n}=\beta_{n} / \epsilon=2 \pi n / \bar{\lambda}$ and $\bar{\lambda}=\epsilon \lambda$ denotes the period for the variable $\xi$. We assume that $\nu=O(\epsilon)$ and introduce an $O(1)$ parameter $\bar{\nu}=\nu / \epsilon$ so that

$$
\widehat{P}_{1}[h] \equiv \sum_{\substack{n=-\infty \\ n \neq 0}}^{\infty} h_{n} P_{w 1}\left(\bar{\beta}_{n} / \bar{v}\right) \mathrm{e}^{\mathrm{i} \bar{\beta}_{n} \xi}, \quad \hat{\tau}_{w 1}[h] \equiv \sum_{\substack{n=-\infty \\ n \neq 0}}^{\infty} h_{n} \tau_{w 1}\left(\bar{\beta}_{n} / \bar{v}\right) \mathrm{e}^{\mathrm{i} \bar{\beta}_{n} \xi} .
$$

\subsection{Long-wave expansion}

We assign the following relative orders between the different parameters and $\epsilon$ : $R e=O(1), \hat{\tau}_{w 0}=O(1)$ and $W e=O\left(\epsilon^{-2}\right)$. We also introduce an $O(1)$ parameter, $\overline{W e}=\epsilon^{2} W e$. For simplicity, let us set $\widehat{P}_{0}^{b}=0$ corresponding to a very wide channel. Next, we expand the streamwise and cross-stream velocities and pressure as follows:

$$
\begin{aligned}
& u=u_{0}+\epsilon u_{1}+\epsilon^{2} u_{2}+\cdots, \\
& w=w_{0}+\epsilon w_{1}+\epsilon^{2} w_{2}+\cdots, \\
& p=p_{0}+\epsilon p_{1}+\epsilon^{2} p_{2}+\cdots .
\end{aligned}
$$

At leading order, we find

$$
u_{0 y y}=-2, \quad p_{0 y}=-2 \cot \theta, \quad u_{0 \xi}+w_{0 y}=0,
$$

subject to

$$
u_{0}=w_{0}=0 \quad \text { at } \quad y=0
$$


and

$$
u_{0 y}=\hat{\tau}_{w 0}, \quad p_{0}=\widehat{P}_{0}^{a}-\overline{W e} \operatorname{Re} h_{\xi \xi}, \quad \text { at } \quad y=h .
$$

The solution of the leading-order problem is

$$
\begin{aligned}
& u_{0}=-y^{2}+2 y h+y \hat{\tau}_{w 0}, \\
& w_{0}=-y^{2} h_{\xi}, \\
& p_{0}=\widehat{P}_{0}^{a}-\bar{W} e \operatorname{Re} h_{\xi \xi}-2 \cot \theta(y-h) .
\end{aligned}
$$

Then, the kinematic boundary condition written as

$$
h_{\tau}+q_{\xi}=0
$$

where $q=\int_{0}^{h} u \mathrm{~d} y$ is the streamwise flow rate, yields

$$
h_{\tau}+\left[\frac{2}{3} h^{3}+\frac{\hat{\tau}_{w 0}}{2} h^{2}\right]_{\xi}+O(\epsilon)=0 .
$$

At next order, we obtain the following system of equations:

$$
\begin{gathered}
u_{1 y y}=\operatorname{Re}\left(u_{0 \tau}+u_{0} u_{0 \xi}+w_{0} u_{0 y}\right)+p_{0 \xi}, \\
p_{1 y}=w_{0 y y}, \\
u_{1 \xi}+w_{1 y}=0,
\end{gathered}
$$

subject to

$$
u_{1}=w_{1}=0, \quad \text { at } \quad y=0,
$$

and

$$
u_{1 y}=\left|\hat{\tau}_{w 0}\right| \bar{\nu} \tau_{w 1}[h], \quad p_{1}=2 w_{0 y}-2 h_{\xi} u_{0 y}+\left|\hat{\tau}_{w 1} \bar{\nu}\right| \widehat{P}_{1}[h] \quad \text { at } \quad y=h,
$$

where $u_{0}, w_{0}$ and $p_{0}$ are given by (4.49)-(4.51). The time derivative $u_{0 \tau}$ involves the time derivative $h_{\tau}$, as is evident from (4.49). The latter is eliminated by using (4.53). The solution of the problem at first order is

$$
\begin{aligned}
u_{1}= & \operatorname{Re}\left(\frac{4}{3} h^{3}-\frac{2}{3} y^{2} h+\frac{1}{6} y^{3}\right) y h h_{\xi}-(\cot \theta)(2 h-y) y h_{\xi}+\operatorname{Re} \overline{W e}\left(h-\frac{1}{2} y\right) y h_{\xi \xi \xi} \\
& +\operatorname{Re} \hat{\tau}_{w 0}\left(\frac{2}{3} h^{3}-\frac{1}{3} y^{2} h+\frac{1}{12} y^{3}\right) y h_{\xi}+\left|\hat{\tau}_{w 0}\right| \bar{v} y \hat{\tau}_{w 1}[h], \\
w_{1}= & -\operatorname{Re}\left(\frac{8}{3} h^{3} h_{\xi}^{2}+\frac{2}{3} h^{4} h_{\xi \xi}-\frac{1}{3} y^{2} h h_{\xi}^{2}-\frac{1}{6} y^{2} h^{2} h_{\xi \xi}+\frac{1}{30} y^{3} h_{\xi}^{2}+\frac{1}{30} y^{3} h h_{\xi \xi}\right) y^{2} \\
& +(\cot \theta)\left(h_{\xi}^{2}+h h_{\xi \xi}-\frac{1}{3} y h_{\xi \xi}\right) y^{2}-\operatorname{Re} \overline{W e}\left(\frac{1}{2} h_{\xi} h_{\xi \xi \xi}+\frac{1}{2} h h_{\xi \xi \xi \xi}-\frac{1}{6} y h_{\xi \xi \xi \xi}\right) y^{2} \\
& -\operatorname{Re} \hat{\tau}_{w 0}\left(h^{2} h_{\xi}^{2}+\frac{1}{3} h^{3} h_{\xi \xi}-\frac{1}{12} y^{2} h_{\xi}^{2}-\frac{1}{12} y^{2} h h_{\xi \xi}+\frac{1}{60} y^{3} h_{\xi \xi}\right) y^{2}-\frac{\left|\hat{\tau}_{w 0}\right| \bar{v}}{2} y^{2} \hat{\tau}_{w 1}\left[h_{\xi}\right], \\
p_{1}= & -2(h+y) h_{\xi}-2 \hat{\tau}_{w 0} h_{\xi}+\left|\hat{\tau}_{w 0}\right| \bar{v} \widehat{P}_{1}[h] .
\end{aligned}
$$

The kinematic condition (4.52) with $q=\int_{0}^{h} u \mathrm{~d} y$ and $u=u_{0}+\epsilon u_{1}+O\left(\epsilon^{2}\right)$, with $u_{0}$ and $u_{1}$ given by (4.49) and (4.59), respectively, yields the following long-wave evolution 
equation at $O(\epsilon)$ :

$$
\begin{aligned}
h_{\tau}+\left[\frac{2}{3} h^{3}+\frac{\hat{\tau}_{w 0}}{2} h^{2}+\epsilon\left(\left[\frac{8 R e}{15} h^{6}-\frac{2 \cot \theta}{3} h^{3}\right] h_{\xi}+\frac{R e \bar{W} e}{3} h^{3} h_{\xi \xi \xi}\right.\right. \\
\left.\left.+\frac{4 R e \hat{\tau}_{w 0}}{15} h^{5} h_{\xi}+\frac{\hat{\tau}_{w 0} \bar{v}}{2} h^{2} \hat{\tau}_{w 1}[h]\right)\right]_{\xi}=0 .
\end{aligned}
$$

Equivalently, using variables $x$ and $t$, we obtain

$$
\begin{aligned}
h_{t}+\left[\frac{2}{3} h^{3}+\frac{\hat{\tau}_{w 0}}{2} h^{2}+\left(\left[\frac{8 R e}{15} h^{6}\right.\right.\right. & \left.-\frac{2 \cot \theta}{3} h^{3}\right] h_{x}+\frac{\operatorname{Re} W e}{3} h^{3} h_{x x x} \\
& \left.\left.+\frac{4 \operatorname{Re} \hat{\tau}_{w 0}}{15} h^{5} h_{x}+\frac{\hat{\tau}_{w 0} v}{2} h^{2} \hat{\tau}_{w 1}[h]\right)\right]_{x}=0,
\end{aligned}
$$

where $\epsilon$ has been scaled out. It is noteworthy that the influence of the normal stress exerted by the gas on the interface, $\widehat{P}_{1}[h]$, turns out to be a higher-order effect.

\subsubsection{Weakly nonlinear evolution}

Let $h=1+\epsilon \eta$. By substituting this expression into (4.62), we obtain the following weakly nonlinear equation:

$$
\begin{aligned}
\eta_{\tau}+\left(2+\hat{\tau}_{w 0}\right) \eta_{\xi}+\epsilon[(4 & \left.+\hat{\tau}_{w 0}\right) \eta \eta_{\xi}+\left(\frac{8 R e}{15}-\frac{2 \cot \theta}{3}+\frac{4 R e \hat{\tau}_{w 0}}{15}\right) \eta_{\xi \xi} \\
& \left.+\frac{\operatorname{Re} \overline{W e}}{3} \eta_{\xi \xi \xi \xi}+\frac{\left|\hat{\tau}_{w 0}\right| \bar{v}}{2}\left(\hat{\tau}_{w 1}[\eta]\right)_{\xi}\right]+O\left(\epsilon^{2}\right)=0 .
\end{aligned}
$$

We write this equation in the moving frame as

$$
\xi=\chi+\left(2+\hat{\tau}_{w 0}\right) \tau
$$

which, after neglecting $O\left(\epsilon^{2}\right)$ terms, becomes

$$
\eta_{\tau}+\epsilon\left(4+\hat{\tau}_{w 0}\right) \eta \eta_{\chi}+\epsilon D \eta_{\chi \chi}+\epsilon \frac{\operatorname{Re} \bar{W} e}{3} \eta_{\chi \chi \chi \chi}+\epsilon \frac{\left|\hat{\tau}_{w 0}\right| \bar{v}}{2}\left(\hat{\tau}_{w 1}[\eta]\right)_{\chi}=0
$$

where $D=D_{1}+4 \operatorname{Re} \hat{\tau}_{w 0} / 15$ and $D_{1}=8 \operatorname{Re} / 15-(2 / 3) \cot \theta$.

The linear stability analysis of the flat solution, $\eta \equiv 0$, leads to the following dispersion relation:

$$
s(k)=-\frac{R e \bar{W} e}{3} k^{4}+D_{1} k^{2}+\frac{4 R e}{15} \hat{\tau}_{w 0} k^{2}-\mathrm{i} k \frac{\left|\hat{\tau}_{w 0}\right| \bar{v}}{2} \tau_{w 1}\left(\frac{k}{\bar{v}}\right) .
$$

We split this relation into its real $(\operatorname{Re}[]$.$) and imaginary (\operatorname{Im}[]$.$) parts as$

$$
\begin{aligned}
& \operatorname{Re}[s(k)]=-\frac{\operatorname{Re} \bar{W} e}{3} k^{4}+D_{1} k^{2}+\frac{4 R e}{15} \hat{\tau}_{w 0} k^{2}+k \frac{\left|\hat{\tau}_{w 0}\right| \bar{v}}{2} \operatorname{Im}\left[\tau_{w 1}\left(\frac{k}{\bar{v}}\right)\right], \\
& \operatorname{Im}[s(k)]=-k \frac{\left|\hat{\tau}_{w 0}\right| \bar{v}}{2} \operatorname{Re}\left[\tau_{w 1}\left(\frac{k}{\bar{v}}\right)\right] .
\end{aligned}
$$

The latter equation implies that the phase velocity is given by

$$
-\frac{\operatorname{Im}[s(k)]}{k}=\frac{1}{2}\left|\hat{\tau}_{w 0}\right| \bar{\nu} \operatorname{Re}\left[\tau_{w 1}\left(\frac{k}{\bar{v}}\right)\right] .
$$


(a)

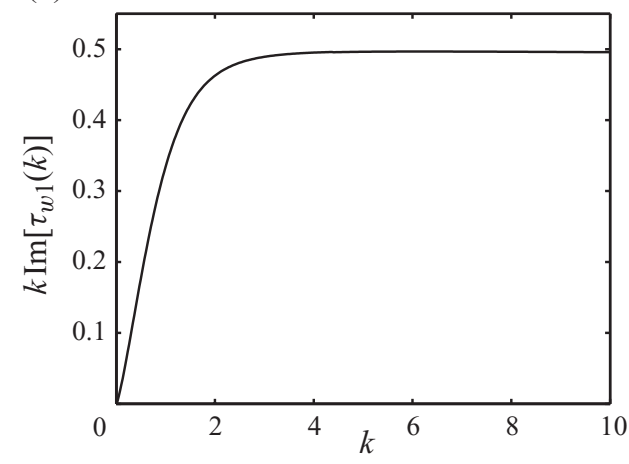

(b)

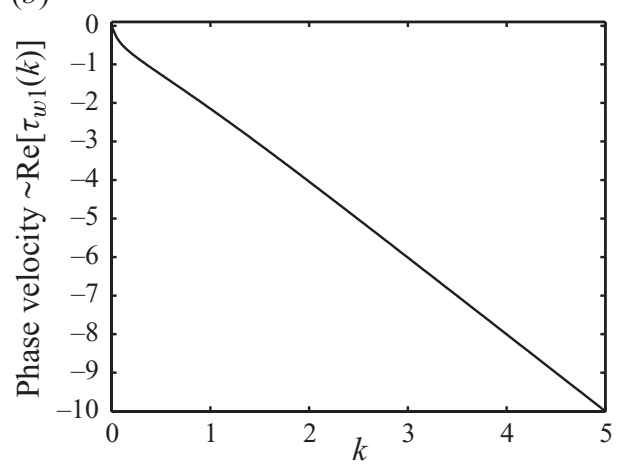

Figure 12. (a) Dependence of the fourth term in the real part of the dispersion relation (4.68) on the wavenumber, $k$. (b) Dependence of $\operatorname{Re}\left[\tau_{w 1}(k)\right]$ on $k$.

Without the gas flow, the trivial solution is stable if $D_{1}<0$, i.e. if $R e<R e_{c} \equiv 5 \cot \theta / 4$. Otherwise, if $D_{1}>0$, i.e. if $R e>R e_{c}$, there is a band of unstable wavenumbers extending from zero. This is a well-known result obtained by Benjamin (1957) and Yih (1963). The effect of the turbulent gas depends on the direction in which the gas flows. For example, for the counter-current case, when $\hat{\tau}_{w 0}<0$, the third term in the dispersion relation (4.67) is quadratic and stabilising, whilst the fourth term is destabilising (for small values of $k$ it increases almost linearly, whilst it approaches a constant value for large values of $k$ ), as is evident from figure 12(a). Therefore, it is apparent that the net effect of these two terms is to introduce a destabilising effect on long waves and a stabilising one on short waves. Also, the gas adds a dispersive effect, as is evident from figure $12(b)$, where $\operatorname{Re}\left[\tau_{w 1}(k)\right]$ is plotted versus $k$.

To simplify (4.66), we introduce new variables

$$
X=\frac{\chi}{A}, \quad H=\frac{\eta}{B}, \quad T=\frac{\tau}{C}
$$

where

$$
A=\sqrt{\frac{\operatorname{Re} \bar{W} e}{3|D|}}, \quad B=\frac{1}{4+\hat{\tau}_{w 0}} \sqrt{\frac{3|D|^{3}}{R e \bar{W} e}}, \quad C=\frac{\operatorname{Re} \bar{W} e}{3 \epsilon D^{2}} .
$$

Equation (4.66) then reduces to the following canonical form:

$$
H_{T}+H H_{X} \pm H_{X X}+H_{X X X X}+\delta\left(\hat{\tau}_{w 1}[H]\right)_{X}=0,
$$

where the $+/-$ sign corresponds to positive/negative value of $D$, respectively, and

$$
\delta=\frac{\left|\hat{\tau}_{w 0}\right| \bar{\nu}}{2\left(4+\hat{\tau}_{w 0}\right) B}=\frac{\left|\hat{\tau}_{w 0}\right| v}{2} \sqrt{\frac{\operatorname{Re} W e}{3|D|^{3}}} .
$$

We note that for

$$
H(X, T)=\sum_{\substack{n=-\infty \\ n \neq 0}}^{\infty} H_{n}(T) \mathrm{e}^{\mathrm{i} \gamma_{n} X}
$$


(a)

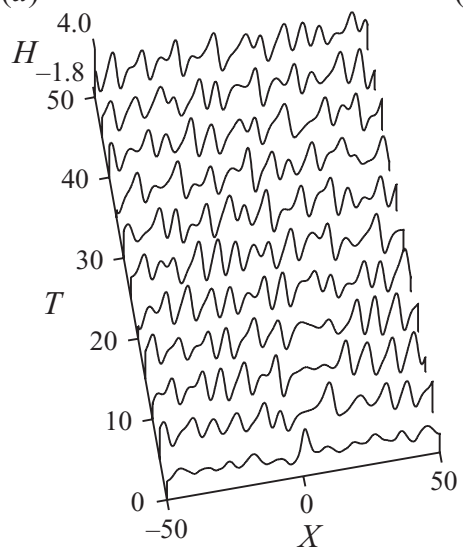

(b)

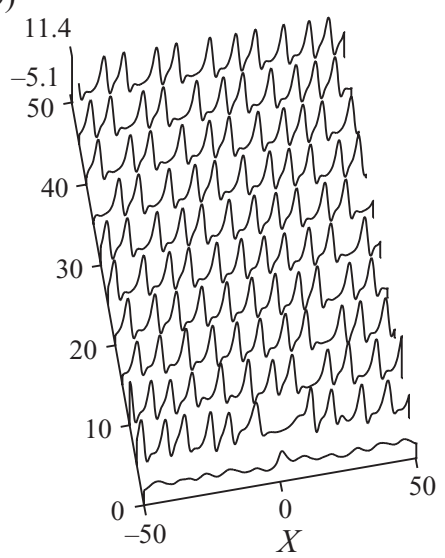

FIGURE 13. Spatio-temporal dynamics for the modified Kuramoto-Sivashinsky equation (4.73) when $(a) \delta=0$ and $(b) \delta=5$. In both cases, the equations are solved on periodic domains of size $[-50,50]$ by a pseudo-spectral method up to $T=50$. The sign in front of the second derivative term is chosen to be positive.

where $\gamma_{n}=2 \pi n / \Lambda$, with $\Lambda=\epsilon \tilde{\lambda} / A h_{0}$ denoting the dimensionless period for the variable $X$, we have

$$
\hat{\tau}_{w 1}[H] \equiv \sum_{\substack{n=-\infty \\ n \neq 0}}^{\infty} H_{n} \tau_{w 1}\left(\frac{\gamma_{n}}{A \bar{v}}\right) \mathrm{e}^{\mathrm{i} \gamma_{n} X} .
$$

In (4.73), the second term is a nonlinear kinematic effect that captures how larger waves move faster than smaller ones, the second term represents instability/energy production with the ' + ' sign and stability/energy dissipation with the '-' sign, at long waves in both cases, the fourth term represents stability/energy dissipation at short waves and the last term corresponds to dispersion.

In fact, (4.73) is a modified Kuramoto-Sivashinsky equation: with the plus sign and $\delta=0$, the equation is the well-known Kuramoto-Sivashinsky equation, first derived independently by Homsy (1974), Lin (1974) and Nepomnyashchy (1974) in the context of a free-falling liquid film. For an extended domain, it is well known that the solution to this equation is spatio-temporal chaos. This is demonstrated in figure 13(a), where a solution of the Kuramoto-Sivashinsky equation is shown on a periodic domain $[-50,50]$ up to $T=50$. The equation was solved by a Fourier pseudo-spectral method with a fourth-order Runge-Kutta integration in time. The destabilising effect of the gas flow could enhance the spatio-temporal complexity of the solution. On the other hand, it is known that dispersion, in the form of a term $\sim H_{X X X}$ (the resulting equation is often referred to as the 'generalised Kuramoto-Sivashinsky equation'), can regularise the solution in favour of a train of spatially periodic cellular structures, each of which approaching a Korteweg-de Vries soliton, see for example Kawahara (1983). The more recent studies by Duprat et al. (2009), Tseluiko et al. (2010a) and Tseluiko, Saprykin \& Kalliadasis (2010b) have analysed further the regularising effect of dispersion for the generalised Kuramoto-Sivashinsky equation and they have formulated a rigorous coherent structures theory to describe the interaction of solitary pulses for sufficiently strong dispersion. Our time-dependent computations with the modified Kuramoto-Sivashinsky equation (4.73) revealed an effect similar to that for 
the generalised Kuramoto-Sivashinsky equation: when dispersive effects dominate, i.e. if the gas parameter $\delta$ is sufficiently large, the solution evolves into an array of travelling pulses. This is illustrated in figure $13(b)$, where a solution is shown for $\delta=5$. The amplitude of the pulses increases if $\delta$ increases.

\subsection{IBL approximation}

We extend the simple Galerkin projection with just one test function (the semiparabolic profile itself) for the free-falling film by Ruyer-Quil \& Manneville (1998, 2000 , 2002) (see also Introduction) to the gas-liquid problem. Assuming that $\epsilon^{2} R e \ll 1$, (4.35) takes the form

$$
p_{y}=-2 \cot \theta+O\left(\epsilon, \epsilon^{2} R e\right) .
$$

Furthermore, if we assume that $\bar{v}$ and $\left|\hat{\tau}_{w 0}\right|$ are $O(1)$ and $\epsilon^{2}$ We $R e \gg \epsilon$, then (4.40) becomes

Therefore,

$$
p=-\epsilon^{2} \text { We Re } h_{\xi \xi}+\hat{P}_{0}^{a}\left|\hat{\tau}_{w 0}\right|+O(\epsilon) \quad \text { at } \quad y=h .
$$

$$
p=2 \cot \theta(h-y)-\epsilon^{2} \text { We Re } h_{\xi \xi}+\hat{P}_{0}^{a}\left|\hat{\tau}_{w 0}\right|+O\left(\epsilon, \epsilon^{2} R e\right) .
$$

Substituting (4.79) into (4.34) and assuming that $R e \gg \epsilon$ and $R e$ is at most $O(1 / \epsilon)$, we obtain

$$
\epsilon \operatorname{Re}\left(u_{\tau}+u u_{\xi}+w u_{y}\right)=-\epsilon\left(2 \cot \theta h_{\xi}-\epsilon^{2} W e \operatorname{Re} h_{\xi \xi \xi}\right)+u_{y y}+2,
$$

where terms $O\left(\epsilon^{2}\right)$ and $O\left(\epsilon^{3} R e\right)$ are neglected. From (4.36) it follows that

$$
w=-\int_{0}^{y} u_{\xi}(\xi, \bar{y}, \tau) \mathrm{d} \bar{y} .
$$

On the wall, we have the no-slip condition

$$
u=0 \quad \text { at } \quad y=0,
$$

while after neglecting $O\left(\epsilon^{2}\right)$ terms, the tangential stress balance condition on the free surface (4.39) becomes

$$
u_{y}=\hat{\tau}_{w}[h]=\hat{\tau}_{w 0}+\epsilon \bar{v}\left|\hat{\tau}_{w 0}\right| \hat{\tau}_{w 1}[h] \quad \text { at } \quad y=h .
$$

Finally, the kinematic-compatibility condition written in conservative form is

$$
h_{\tau}+q_{\xi}=0,
$$

where $q=\int_{0}^{h} u \mathrm{~d} y$ is the streamwise flow rate. Equations (4.80)-(4.84) are the so-called first-order boundary-layer equations.

We now project the velocity field onto polynomial test functions

$$
u=\sum_{i=1}^{N} a_{i}(\xi, \tau) \eta^{i},
$$

where $\eta=y / h(\xi, \tau)$ is a similarity variable. This expansion automatically satisfies the no-slip condition at the wall. We require that the integral of this expansion with respect to $y$ from 0 to $h(\xi, \tau)$ gives the flow rate $q$ in the streamwise direction, which leads to

$$
\frac{a_{1}}{2}+\frac{a_{2}}{3}=\frac{q}{h}-\sum_{i=3}^{N} \frac{a_{i}}{i+1} .
$$


We also require that the interfacial boundary condition (4.83) is satisfied, which gives

$$
a_{1}+2 a_{2}=h \hat{\tau}_{w}[h]-\sum_{i=3}^{N} i a_{i} .
$$

Solving (4.86) and (4.87) for $a_{1}$ and $a_{2}$, we find

$$
\begin{aligned}
& a_{1}=3 \frac{q}{h}-\frac{1}{2} h \hat{\tau}_{w}[h]+\sum_{i=3}^{N}\left(\frac{i}{2}-\frac{3}{i+1}\right) a_{i}, \\
& a_{2}=-\frac{3}{2} \frac{q}{h}+\frac{3}{4} h \hat{\tau}_{w}[h]+\sum_{i=3}^{N}\left(\frac{3}{2(i+1)}-\frac{3 i}{4}\right) a_{i} .
\end{aligned}
$$

In effect, the elimination of $a_{1,2}$ 'homogenises' the tangential stress boundary condition and is equivalent to a tau method (see also Kalliadasis et al. 2003a,b). Substituting the above expressions for $a_{1}$ and $a_{2}$ into (4.85) yields

$$
u=u^{(0)}+u^{(1)}+\sum_{i=2}^{N-1} a_{i+1} \phi_{i}(\eta)
$$

where

$$
u^{(0)}=3 \frac{q}{h}\left(\eta-\frac{1}{2} \eta^{2}\right), \quad u^{(1)}=-\frac{1}{2} h \hat{\tau}_{w}[h]\left(\eta-\frac{3}{2} \eta^{2}\right)
$$

and also,

$$
\phi_{i}(\eta)=\left(\frac{i+1}{2}-\frac{3}{i+2}\right) \eta+\frac{3}{4}\left(\frac{2}{i+2}-i-1\right) \eta^{2}+\eta^{i+1}
$$

Expansion (4.90) can also be written as

$$
u=u^{(1)}+\sum_{i=1}^{N-1} b_{i} \phi_{i}(\eta)
$$

where $\phi_{1}(\eta)=\eta-\eta^{2} / 2$ and $b_{1}=3 q / h, b_{i}=a_{i+1}$ for $i=2, \ldots, N-1$.

In general, $N$ should be sufficiently large to achieve convergence. However, like the free-falling film problem, it is sufficient to take $N=2$ and use a Galerkin projection with one test function, $\phi_{1}$, in order to obtain a model fully resolving the behaviour close to criticality and to describe satisfactorily the nonlinear regime. Thus, we substitute

$$
u=u^{(1)}+b_{1} \phi_{1}(\eta)=u^{(0)}+u^{(1)}
$$

into (4.34) to obtain the following residual:

$$
\begin{aligned}
\mathscr{R}= & \epsilon \operatorname{Re}\left[u_{\tau}^{(0)}+u_{a \tau}^{(1)}+\left(u^{(0)}+u_{a}^{(1)}\right)\left(u_{\xi}^{(0)}+u_{a \xi}^{(1)}\right)+w^{(0)}\left(u_{y}^{(0)}+u_{a y}^{(1)}\right)\right] \\
& +\epsilon\left[2 \cot \theta h_{\xi}+\epsilon^{2} \text { We Re } h_{\xi \xi \xi}\right]-u_{y y}^{(0)}-u_{a y y}^{(1)}-u_{b y y}^{(1)}-2+O\left(\epsilon^{2} \operatorname{Re}\right),
\end{aligned}
$$

where

$$
u_{a}^{(1)}=-\frac{1}{2} \hat{\tau}_{w 0} h\left(\eta-\frac{3}{2} \eta^{2}\right), \quad u_{b}^{(1)}=-\frac{1}{2} \epsilon \bar{v}\left|\hat{\tau}_{w 0}\right| h \hat{\tau}_{w 1}[h]\left(\eta-\frac{3}{2} \eta^{2}\right)
$$


and

$$
w^{(0)}=-\int_{0}^{y}\left[u_{\xi}^{(0)}(\xi, \bar{y}, \tau)+u_{a \xi}^{(1)}(\xi, \bar{y}, \tau)\right] \mathrm{d} \bar{y} .
$$

We assume that $\epsilon R e \ll 1$ (which gives a tighter upper bound on $R e$ compared to the requirement $\epsilon^{2} R e \ll 1$ for the boundary-layer equations) and we ignore terms $O\left(\epsilon^{2} R e\right)$ in (4.95). Next, we require that the residual is orthogonal to a weight function $w_{1}$,

$$
\left\langle\mathscr{R}, w_{1}\right\rangle \equiv \int_{0}^{1} \mathscr{R} w_{1} \mathrm{~d} \eta=0 .
$$

In the case of the Galerkin method, $w_{1}=\phi_{1}$. Then, (4.98) yields the following partial differential equation:

$$
\begin{aligned}
& q_{\tau}=-\frac{17}{7} \frac{q q_{\xi}}{h}+\frac{9}{7} \frac{q^{2} h_{\xi}}{h^{2}}-\frac{5}{2 \epsilon R e} \frac{q}{h^{2}}+\frac{5}{3 \epsilon R e} h-\frac{5 \cot \theta}{3 R e} h h_{\xi}+\frac{5 \epsilon^{2} W e}{6} h h_{\xi \xi \xi} \\
& +\hat{\tau}_{w 0}\left(\frac{5}{4 \epsilon \operatorname{Re}}-\frac{19 \hat{\tau}_{w 0}}{672} h h_{\xi}-\frac{19}{336} h q_{\xi}-\frac{5}{112} q h_{\xi}+\frac{5 \operatorname{sign}\left(\hat{\tau}_{w 0}\right) \bar{v}}{4 R e} \hat{\tau}_{w 1}[h]\right),
\end{aligned}
$$

where $h_{\tau}$ has been replaced by $-q_{\xi}$ by using the kinematic compatibility (4.84). Equation (4.99) together with (4.84) is the IBL approximation for a falling film in contact with a turbulent gas. By writing these equations using $x$ and $t$ variables, we eliminate the film parameter $\epsilon$ :

$$
\begin{gathered}
h_{t}+q_{x}=0 \\
q_{t}=-\frac{17}{7} \frac{q q_{x}}{h}+\frac{9}{7} \frac{q^{2} h_{x}}{h^{2}}-\frac{5}{2 R e} \frac{q}{h^{2}}+\frac{5}{3 R e} h-\frac{5 \cot \theta}{3 R e} h h_{x}+\frac{5 W e}{6} h h_{x x x} \\
+\hat{\tau}_{w 0}\left(\frac{5}{4 R e}-\frac{19 \hat{\tau}_{w 0}}{672} h h_{x}-\frac{19}{336} h q_{x}-\frac{5}{112} q h_{x}+\frac{5 \operatorname{sign}\left(\hat{\tau}_{w 0}\right) v}{4 R e} \hat{\tau}_{w 1}[h]\right) .
\end{gathered}
$$

The terms in the second line of (4.101) correspond to the influence of the gas. When the gas shear-strength parameter $\Theta$ vanishes, $\hat{\tau}_{w 0}$ also vanishes and (4.100) and (4.101) reduce to the 'first-order model' of Ruyer-Quil \& Manneville $(1998,2000,2002)$ for a free-falling film. Note that the influence of the normal stress exerted by the gas on the interface, $\widehat{P}_{1}[h]$, turns out to be a higher-order effect, as in the long-wave model (4.63), and should not be taken into account, in contrast to the work by Demekhin (1981).

Let us now demonstrate that the first-order long-wave equation (4.62) can be recovered from an appropriate expansion of our IBL system. For this purpose, we use the variables $\xi$ and $\tau$ and assume that $W e=\overline{W e} / \epsilon^{2}$, where $\overline{W e}=O(1)$. Then, we expand $q$ as $q=q_{0}+\epsilon q_{1}+\cdots$. From (4.99), we obtain at leading order

$$
q_{0}=\frac{2}{3} h^{3}+\frac{1}{2} \hat{\tau}_{w 0} h^{2} .
$$

At next order, we find

$$
\begin{aligned}
q_{1}=\frac{2 R e}{5} & h^{2}\left(-q_{0 \tau}-\frac{17}{7} \frac{q_{0} q_{0 \xi}}{h}+\frac{9}{7} \frac{q_{0}^{2} h_{\xi}}{h^{2}}-\frac{5}{3} \frac{\cot \theta}{R e} h h_{\xi}+\frac{5}{6} \overline{W e} h h_{\xi \xi \xi}\right. \\
& \left.+\hat{\tau}_{w 0}\left[-\frac{19}{672} \hat{\tau}_{w 0} h^{2} h_{\xi}-\frac{19}{336} q_{0 \xi} h-\frac{5}{112} q_{0} h_{\xi}+\frac{5 \bar{v}}{4 R e} \hat{\tau}_{w 1}[h]\right]\right) .
\end{aligned}
$$


Substituting (4.102) into (4.103) yields

$$
\begin{aligned}
q_{1}=- & \frac{4 R e}{5} h^{4} h_{\tau}-\frac{16 R e}{15} h^{6} h_{\xi}-\frac{2 \cot \theta}{3} h^{3} h_{\xi}+\frac{R e \bar{W} e}{3} h^{3} h_{\xi \xi \xi} \\
& -\frac{2 \hat{\tau}_{w 0} R e}{5} h^{3} h_{\tau}-\frac{4 \hat{\tau}_{w 0} R e}{3} h^{5} h_{\xi}-\frac{2 \hat{\tau}_{w 0}^{2} \operatorname{Re}}{5} h^{4} h_{\xi}+\frac{\hat{\tau}_{w 0} \bar{v}}{2} h^{2} \hat{\tau}_{w 1}[h] .
\end{aligned}
$$

Replacing $h_{\tau}$ with $-q_{0 \xi}+O(\epsilon)$ and using $q_{0}$ from (4.102), we obtain

$$
q_{1}=\left[\frac{8 R e}{15} h^{6}-\frac{2 \cot \theta}{3} h^{3}\right] h_{\xi}+\frac{R e \bar{W} e}{3} h^{3} h_{\xi \xi \xi}+\frac{4 \hat{\tau}_{w 0} R e}{15} h^{5} h_{\xi}+\frac{\hat{\tau}_{w 0} \bar{v}}{2} h^{2} \hat{\tau}_{w 1}[h]+O(\epsilon) .
$$

Equation (4.84) with $q=q_{0}+\epsilon q_{1}$, where $q_{0}$ and $q_{1}$ are given by (4.102) and (4.105), respectively, yields the long-wave equation (4.62). Hence, our IBL model fully resolves the behaviour close to criticality.

\section{Nonlinear waves}

\subsection{Travelling waves}

Here, we investigate travelling-wave solutions of our IBL model. Such solutions are known to exist in the case of free-falling films. We begin by writing (4.100) and (4.101) in a frame moving with the velocity $c$ of a travelling wave. For steady solutions in this frame, we have the system of equations:

$$
\begin{gathered}
-c h_{x}+q_{x}=0, \\
-c q_{x}=-\frac{17}{7} \frac{q q_{x}}{h}+\frac{9}{7} \frac{q^{2} h_{x}}{h^{2}}-\frac{5}{2 \operatorname{Re}} \frac{q}{h^{2}}+\frac{5}{3 \operatorname{Re}} h-\frac{5 \cot \theta}{3 \operatorname{Re}} h h_{x}+\frac{5 W e}{6} h h_{x x x} \\
+\hat{\tau}_{w 0}\left(\frac{5}{4 R e}-\frac{19 \hat{\tau}_{w 0}}{672} h h_{x}-\frac{19}{336} h q_{x}-\frac{5}{112} q h_{x}+\frac{5 \operatorname{sign}\left(\hat{\tau}_{w 0}\right) v}{4 R e} \hat{\tau}_{w 1}[h]\right) .
\end{gathered}
$$

This system is solved on a periodic domain with a numerical scheme based on spectral representation of the derivatives and Newton iterations to obtain solutions from initial guesses. In our computations, we fix the volume of the liquid in one period so that the undisturbed film thickness is 1 . We restrict our attention to single-hump waves, the so-called $\gamma_{2}$ waves (see, for example, Chang, Demekhin \& Kopelevich 1993; Chang \& Demekhin 2002), which are the main feature of the evolution following destabilisation of the flat base flow in the case of a free-falling film. In the limit of the wave period tending to infinity, such waves converge to one-hump solitary waves that travel faster than infinitesimally small waves. The flow downstream can be described as a superposition of such near-solitary waves.

We use a counter-current helium-methanol flow as a working system. Figure 14 shows the dependence of the velocity, $c$, of periodic travelling waves as a function of the Reynolds number, $R e$, for various values of the gas shear-stress parameter, $\Theta$. The travelling waves are computed in a periodic domain whose half is 91.96. This equals to the period of the most unstable wave for $R e=5$, when there is no gas flow above the liquid film. As the gas shear-stress parameter, $\Theta$, is increased, the velocity of the waves decreases. Note that the curve obtained for $\Theta=0$ is qualitatively similar to that computed by Ruyer-Quil \& Manneville (2000, figure 4) using a mixture of glycerol and water as the working fluid. The numerical results are obtained with a pseudo-arclength continuation procedure. In figures 15 and 16 we plot the dependence 


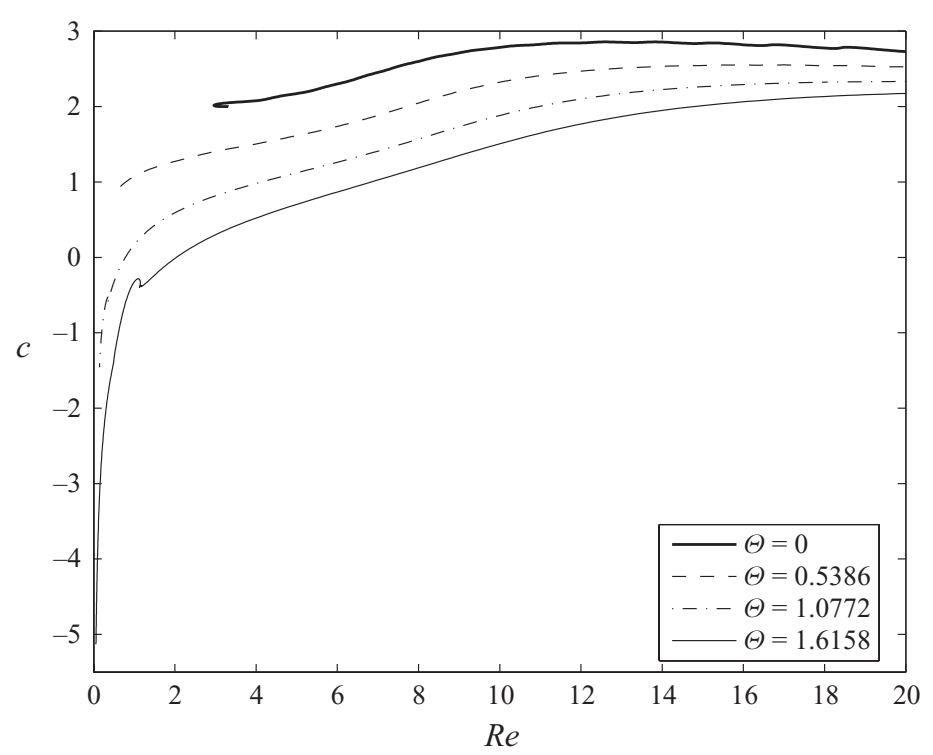

FIGURE 14. Dependence of the velocity, $c$, of periodic travelling waves on the Reynolds number, $R e$, for various values of the gas shear-stress parameter, $\Theta$, obtained from the IBL model. The working system is helium-methanol. The half-length of the periodic domain is 91.96, which equals to the period of the most unstable wave for $R e=5$ when there is no gas flow above the liquid film.

of the maximum and the minimum, respectively, of the wave height on the Reynolds number, $R e$, for various values of the gas shear-stress parameter, $\Theta$. For non-zero $\Theta$, both the maximum and the minimum approach unity (i.e. the solution converges to a flat solution) as $R e$ tends to a certain value that depends on $\Theta$. Note that if $\Theta$ is sufficiently large (see the curves for $\Theta=1.0772$ and 1.6158), then the maximum and minimum curves exhibit local minima, maxima and turning points.

Figure 17 shows wave profiles for $R e=5$ as the gas friction velocity is increased. Figures 18-20 depict the dependence of the velocity, the minimum and the maximum of the periodic travelling wave as functions of the gas friction velocity for several values of the Reynolds number, $R e=5,7$ and 9. The computations are done on a periodic domain whose half-length is equal to the period of the most unstable wave. As the gas friction velocity increases, the travelling wave velocity decreases and at the same time the maximum height of the wave grows, whilst the minimum value decreases. At a certain value of the gas friction velocity, $U_{f}^{*}$, a large-amplitude standing wave $(c=0)$ forms, which should then correspond to the onset of flooding. The dependence of the gas friction velocity corresponding to flooding, $U_{f}^{*}$, on the Reynolds number is depicted in figure 21. As the Reynolds number increases, the velocity $U_{f}^{*}$ decreases, which is consistent with experimental findings, e.g. by Semyonov (1944) described in the Introduction. It should be noted that the results are sensitive to the period. This is to be expected and was also found by Trifonov (2010). It suggests that the flooding velocity is sensitive to the typical wave-separation length. It should also be noted that we refrain from comparing quantitatively our results with experiments since our model is two-dimensional, unlike the experiments which are often done in three-dimensional geometries, i.e. tubes (the experiments used for the comparison with our theoretical predictions for the gas phase in $\S 3.2 .1$ were 


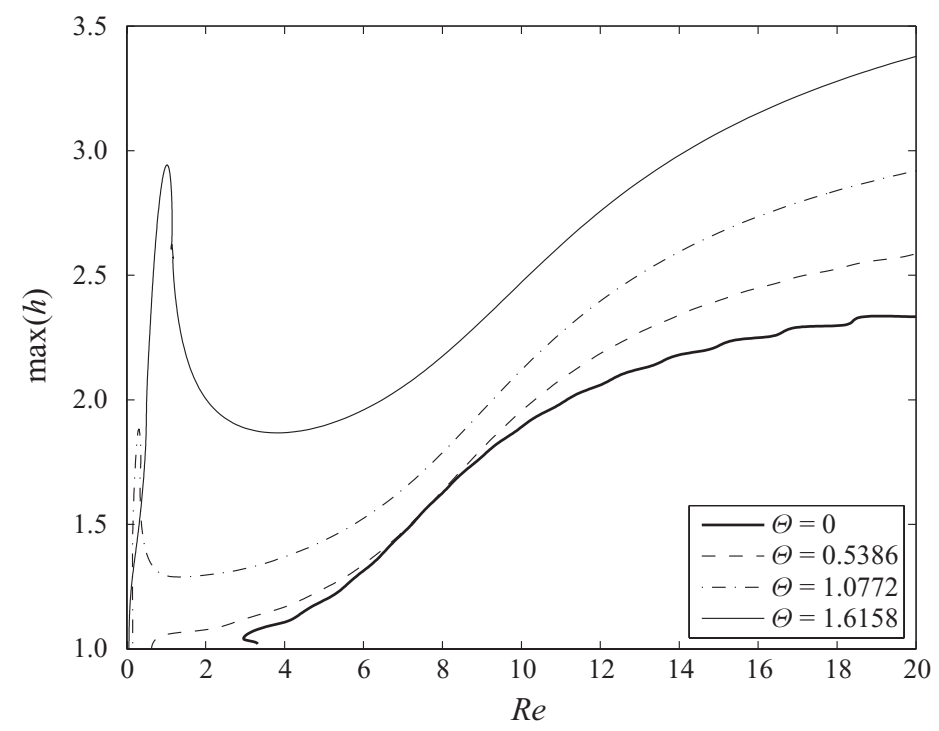

FIGURE 15. Maximum height of the periodic travelling waves computed in figure 14 as a function of the Reynolds number, $R e$.

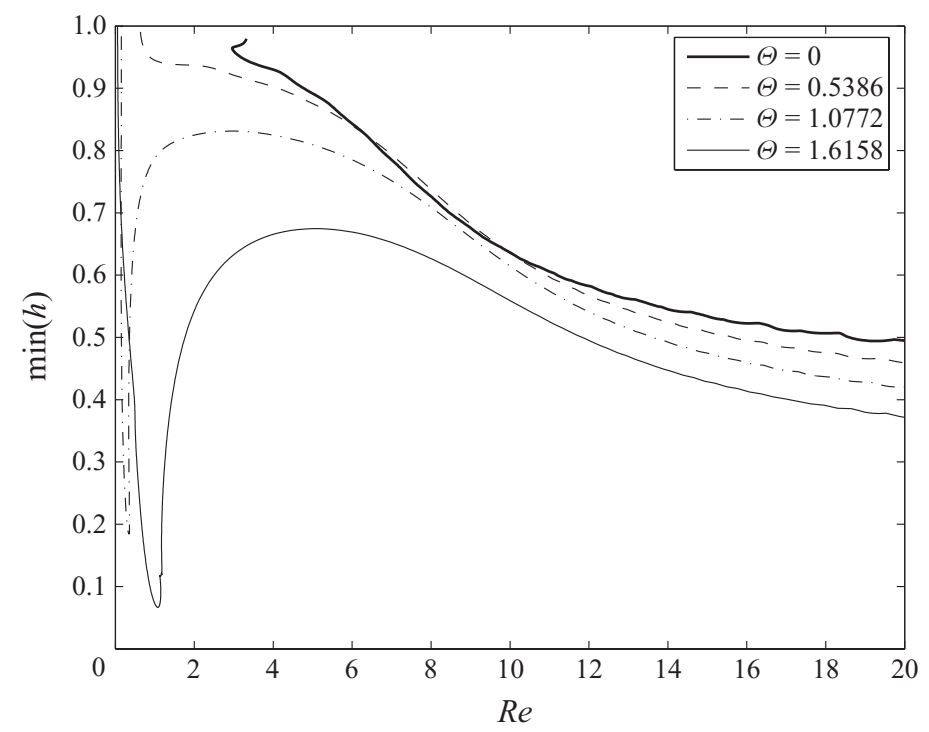

FIGURE 16. Minimum height of the periodic travelling waves computed in figure 14 as a function of the Reynolds number, $R e$.

done in wide channels and hence the corresponding geometries can be considered as two-dimensional). A noted exception is the study by Drosos et al. (2006) with a channel but for much larger $\operatorname{Re}(\sim 100)$. Nevertheless, we find qualitative agreement with experiments, and the developed framework allows for a systematic theoretical investigation of the onset of flooding. A three-dimensional study is left as a topic for future research.

Once again, we have analysed the influence of a turbulent gas only on the $\gamma_{2}$ waves, because such waves are the main feature of the evolution of the system following 


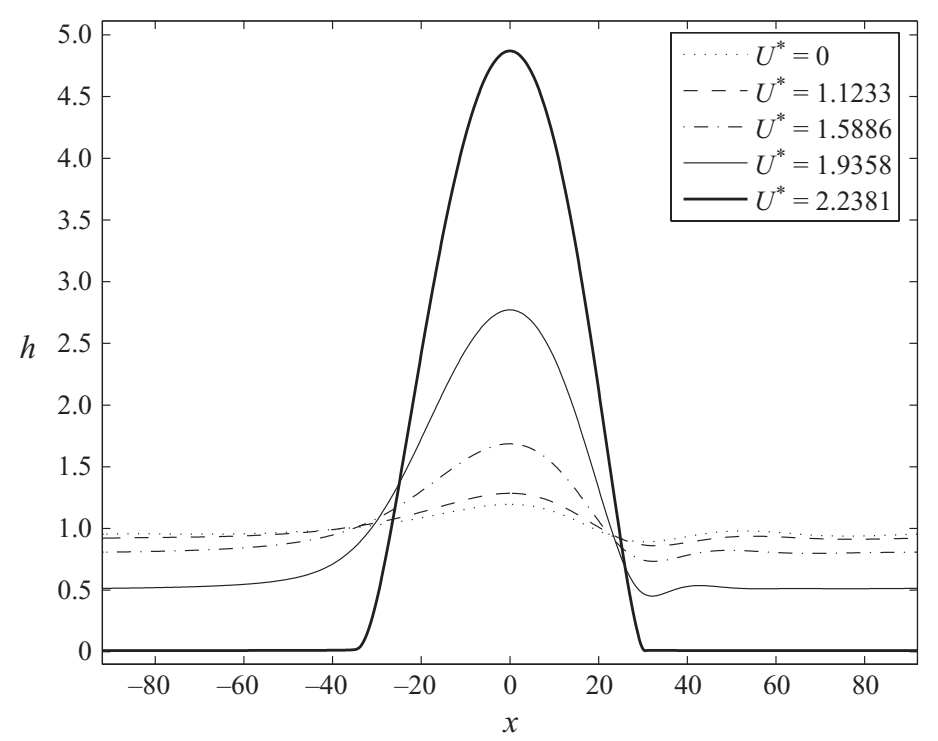

FIGURE 17. Periodic travelling waves for helium-methanol flows obtained from the IBL model when $R e=5$ and the gas friction velocity is increased.

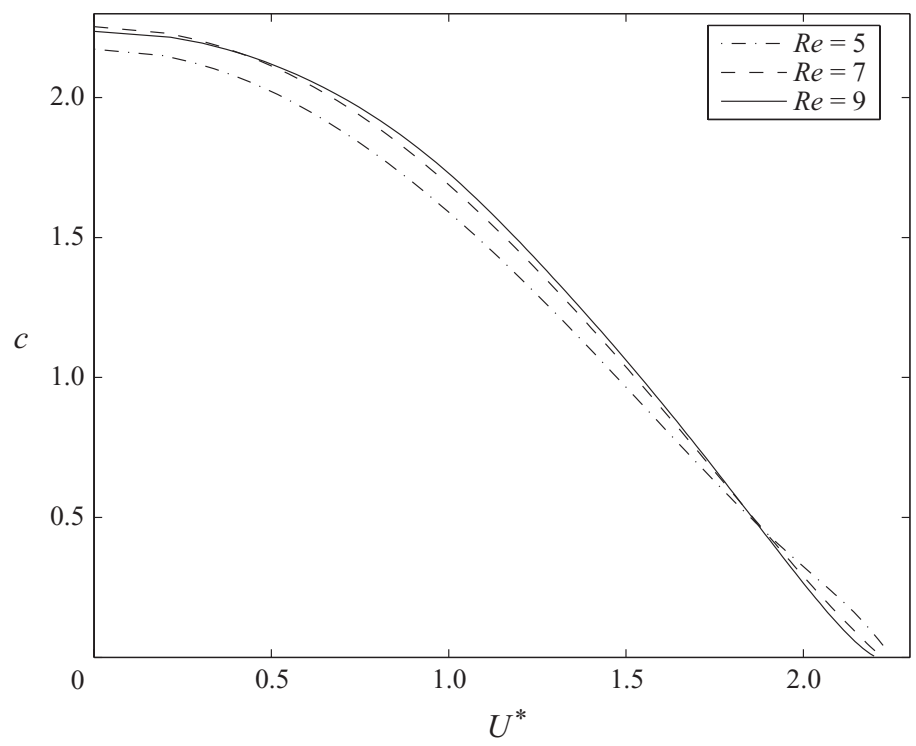

FIGURE 18. Dependence of the velocity, $c$, of a periodic travelling wave on the gas friction velocity, $U^{*}$, for various values of the Reynolds number, $R e$, obtained from the IBL model. The working system consists of helium and methanol. The half-length of the periodic domain equals to the period of the most unstable wave when there is no gas flow above the liquid film, i.e. $U^{*}$ vanishes.

the destabilisation of the flat solution. However, there must exist other wave families as in the free-falling film problem, for example, Chang et al. (1993), Ruyer-Quil \& Manneville (2000), Chang \& Demekhin (2002) and Meza \& Balakotaiah (2008). Let us consider the influence of the turbulent gas on the so-called $\gamma_{1}$ waves, for instance. For the IBL model we have derived, these waves are near-sinusoidal ones for the case 


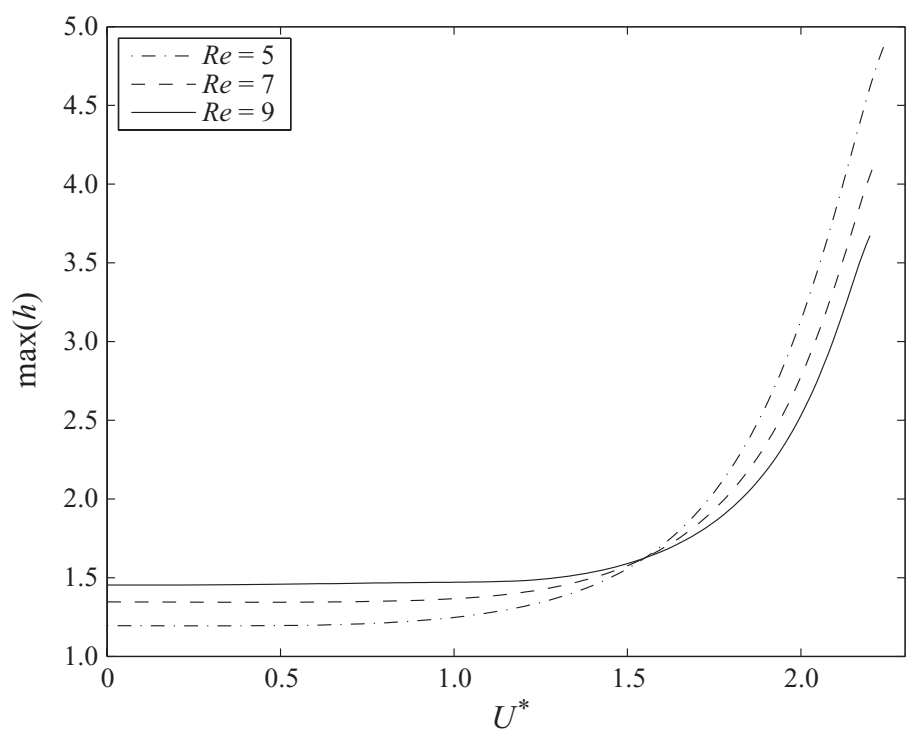

FIGURE 19. Dependence of the maximum height of a periodic travelling wave on the gas friction velocity, $U^{*}$, for the waves computed in figure 18 .

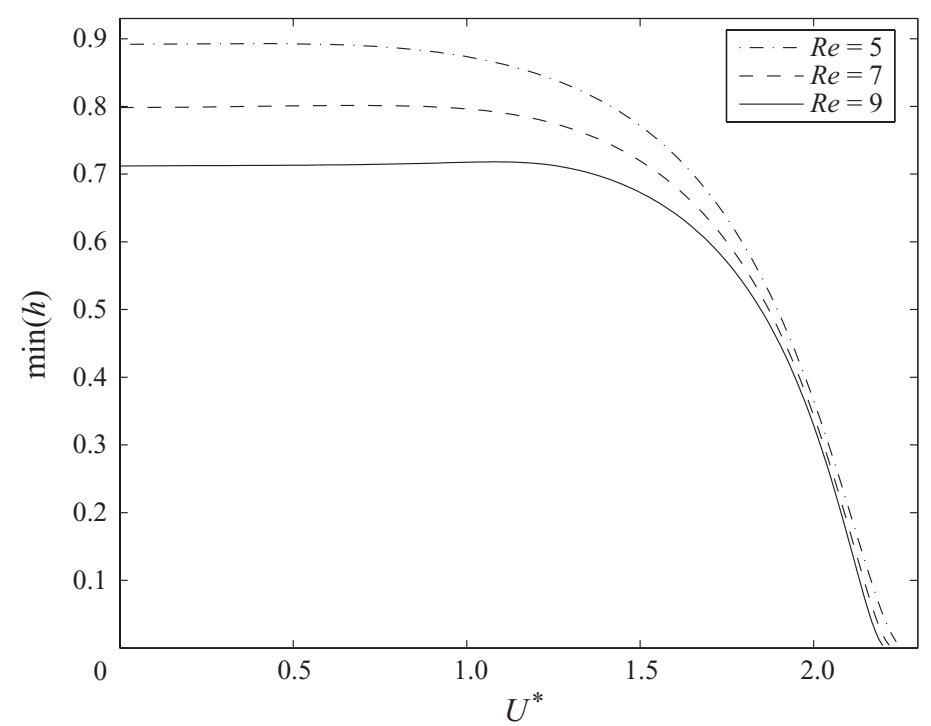

FIGURE 20. Dependence of the minimum of a periodic travelling wave on the gas friction velocity $U^{*}$, for the waves computed in figure 18 .

when the wave period is just above the instability threshold, $\pi \sqrt{5 W e / 2}$, and approach one-hollow inverted solitary waves when the period tends to infinity. Figures 22-24 depict the dependence of the velocity, the minimum and the maximum of the periodic travelling waves as functions of the gas friction velocity for $R e=5$. The computations have been performed in a periodic domain whose half-length is equal to the period of the most unstable wave. A pseudo-arclength continuation procedure has been used to produce the curves. The circles in the figures correspond to a $\gamma_{1}$ wave and indicate the 


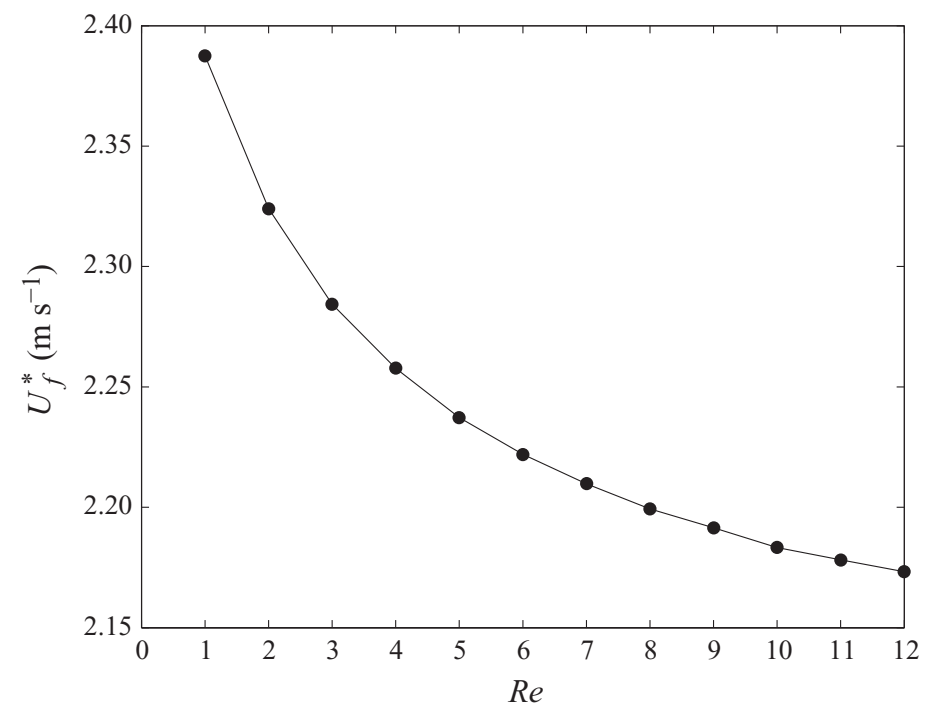

FIGURE 21. Dependence of the gas friction velocity corresponding to the onset of flooding, $U_{f}^{*}$, on the Reynolds number, Re. At each value of the Reynolds number, the computations are done in a periodic domain equal twice the period of the most unstable wave when $U^{*}$ vanishes.

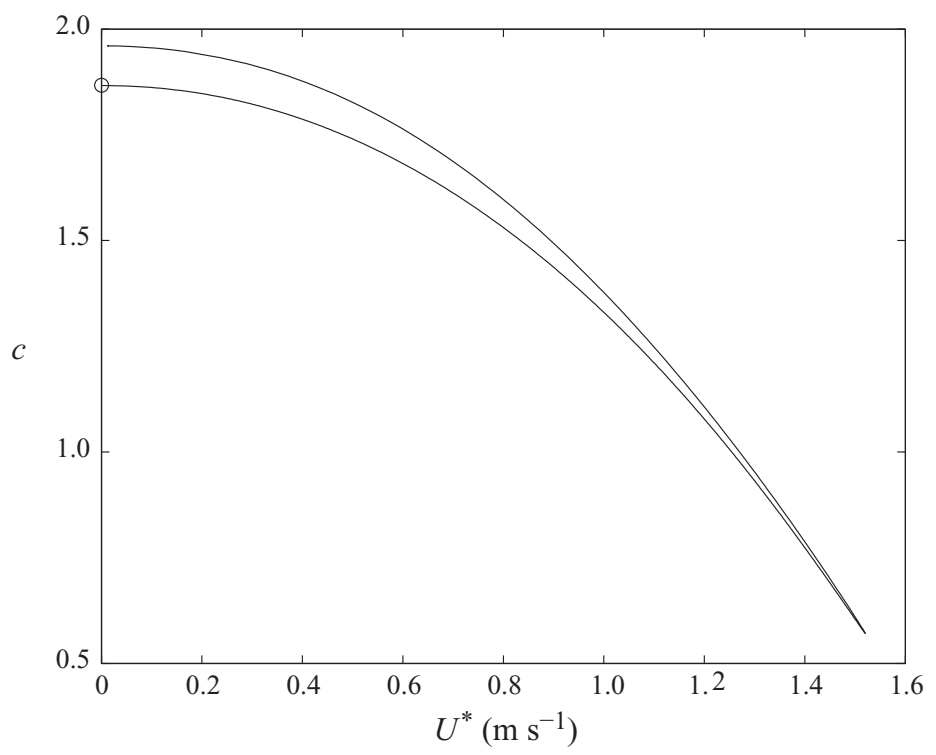

FIGURE 22. Dependence of the velocity, $c$, of a periodic travelling wave on the gas friction velocity, $U^{*}$, for $R e=5$ obtained from the IBL model. The working system consists of helium and methanol. The half-length of the periodic domain equals to the period of the most unstable wave when there is no gas flow above the liquid film, i.e. $U^{*}$ vanishes. The circle corresponds to a $\gamma_{1}$ wave and indicates the point at which the continuation starts.

point at which the continuation was started. As is evident from the figures, the gas friction velocity cannot be increased beyond a certain critical value $\left(U^{*} \approx 1.52 \mathrm{~m} \mathrm{~s}^{-1}\right)$ for the computed branch. The curves have turning points, and the continuation ends up at $U^{*}=0 \mathrm{~m} \mathrm{~s}^{-1}$, connecting the $\gamma_{1}$ branch with another branch. We have checked 


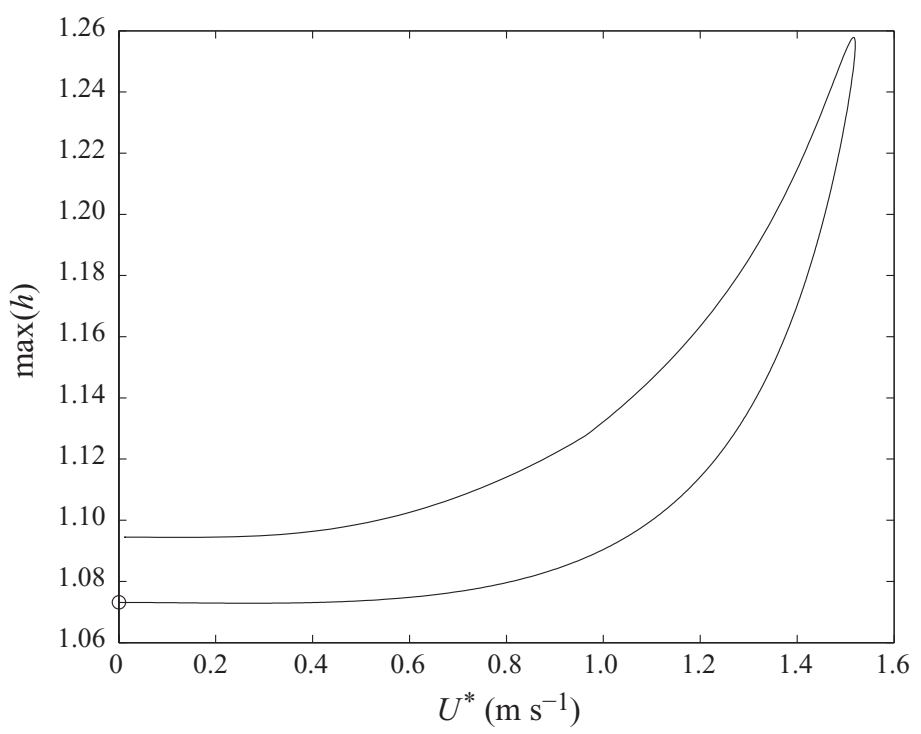

FIGURE 23. Dependence of the maximum height of a periodic travelling wave on the gas friction velocity, $U^{*}$, for the waves computed in figure 22 .

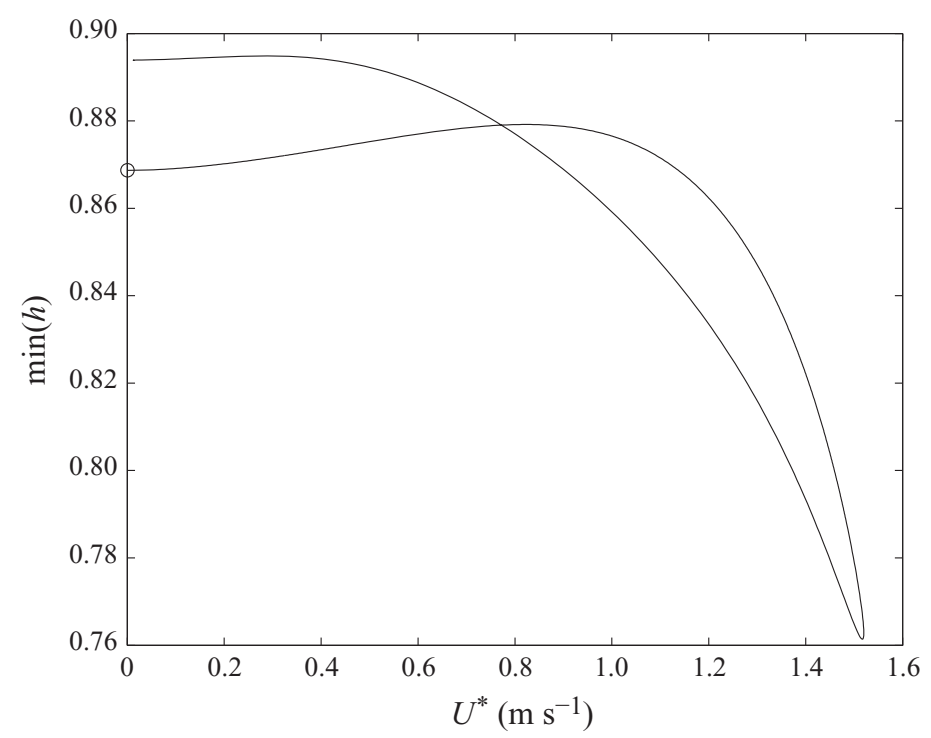

FIGURE 24. Dependence of the minimum of a periodic travelling wave on the gas friction velocity $U^{*}$, for the waves computed in figure 22 .

that the waves of this branch approach a two-hollow inverted solitary wave when the wave period tends to infinity. Figure 25 shows the wave profile at the start of the continuation procedure (the $\gamma_{1}$ wave displayed as a solid line), at the maximal value of the gas friction velocity (dot-dashed line) and at the end of the continuation procedure (dashed line). The analysis of the influence of the turbulent gas on other wave families is left as a topic for further investigation.

Finally, we note that recent literature shows the existence of flow reversal in falling liquid films, e.g. Malamataris \& Balakotaiah (2008), Dietze, Leefken \& Kneer (2008) 


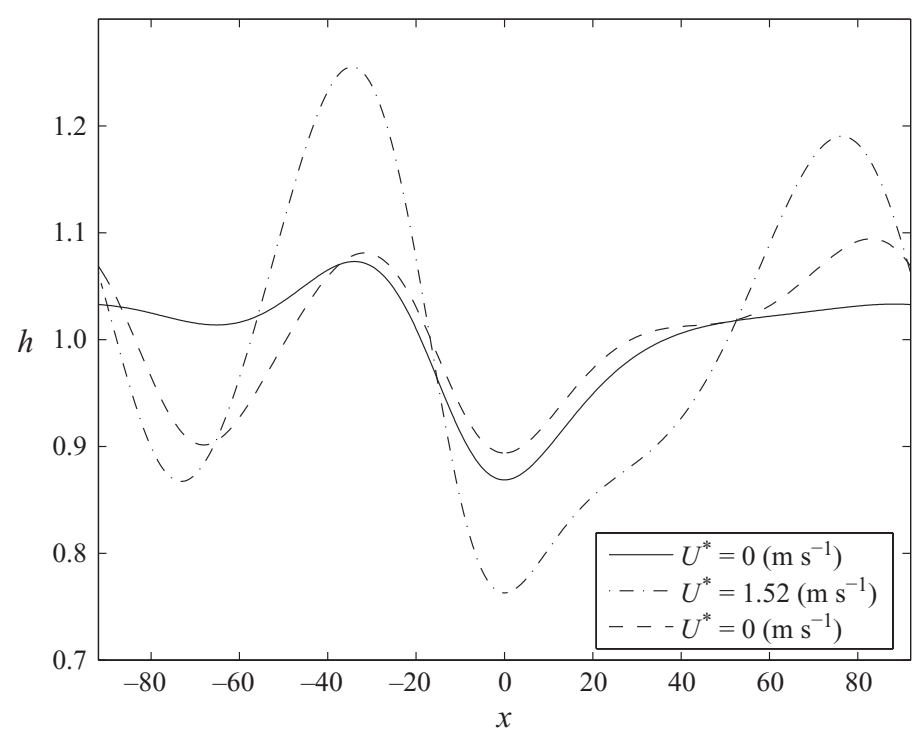

FIGURE 25. Periodic travelling waves for helium-methanol flows obtained from the IBL model when $R e=5$ and the gas friction velocity is increased. The solid line is the $\gamma_{1}$ wave $\left(U^{*}=0 \mathrm{~m} \mathrm{~s}^{-1}\right)$, the dashed line shows the wave corresponding to the maximum gas friction velocity, $U^{*}=1.35 \mathrm{~m} \mathrm{~s}^{-1}$, obtained in figure 22 , and the dot-dashed line corresponds to the final wave profile computed in figure 22 at which the continuation procedure stopped $\left(U^{*}=0 \mathrm{~m} \mathrm{~s}^{-1}\right)$.

and Dietze, Al-Sibai \& Kneer (2009). In these studies, the value of the Reynolds number was taken to be sufficiently far away from its critical value, e.g. in the study of Malamataris \& Balakotaiah (2008), the value of the Reynolds number was 18.6 (according to our definition of the Reynolds number) and the value of the Kapitza number was 202.27, and then a roll formation was observed below a minimum of the wave. The larger the Kapitza number is, the more rolls are present, e.g. for the Kapitza number 1000 three regions of flow reversal were observed, but we should note that at the same time the value of the Reynolds number was taken to be approximately 45 , which is well beyond the range we considered in our work. Our results are mainly relevant to the case of the Reynolds number not too far away from its critical value, implying large values of the Weber number, where flow reversal is not so significant or not present at all (see, for example, figure 1 in Meza \& Balakotaiah 2008).

\subsection{Time-dependent evolution}

To examine the spatio-temporal dynamics of the film, we must numerically solve the system (4.100) and (4.101) as an initial-value problem. This will also allow us to address the question of relevance of large-amplitude standing wave solutions we computed earlier, which is related to the way such solutions attract initial conditions.

To solve (4.100) and (4.101) as an initial-value problem, we have used a fully implicit time-dependent scheme based on a Fourier pseudo-spectral representation of the derivatives together with fast Fourier transforms. To advance the solution dynamically from the time level $n$ to the time level $n+1$, the system of nonlinear equations for the unknown solution at the new time level, $n+1$, is solved iteratively using Newton's method. Typical results of our computations are shown in figure 26 for $R e=5$. First, the evolution of the film is computed using as initial condition random noise of amplitude 0.05 in the absence of a turbulent gas up to $t=10000$ 
(a)

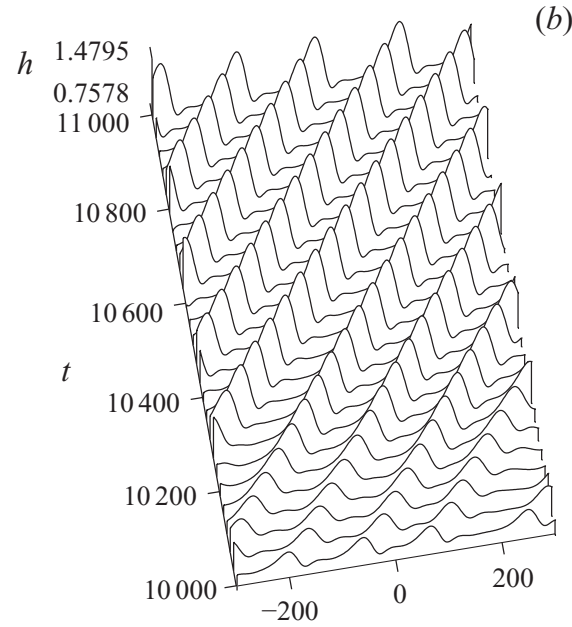

(c)

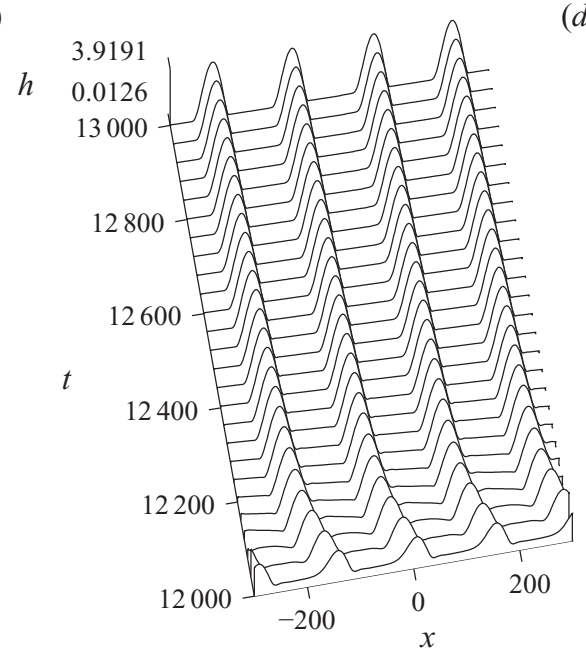

(b)

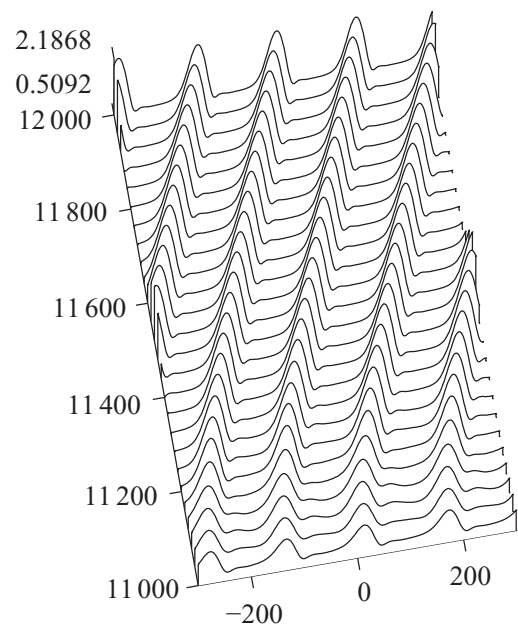

(d)

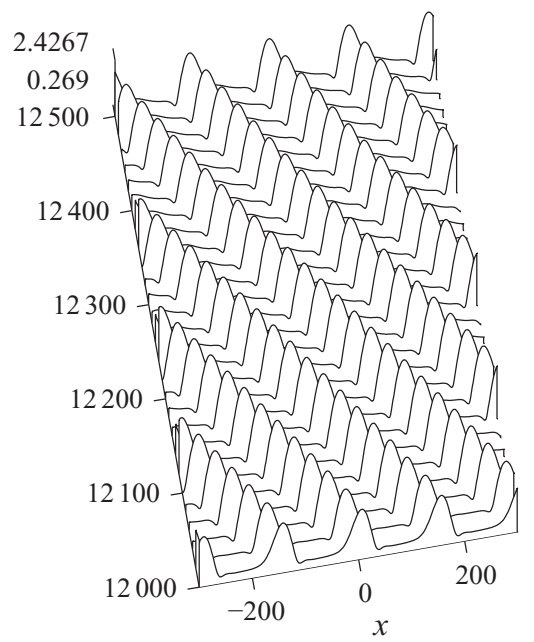

FIGURE 26. Time evolution of the gas-liquid interface. Plots $(a-d)$ correspond to $\Theta=1.26,1.89,2.52$ and 3.15 , respectively, or, equivalently, to $U^{*}=1.50,1.83,2.11$ and $2.36 \mathrm{~m} \mathrm{~s}^{-1}$, respectively. The computations are performed on the periodic domain $[-300,300]$. The initial condition for $(a)$ is the solution obtained by using a random noise for the case when the turbulent gas is absent and integrating up to $t=10000$. The initial condition for $(b)$ is the solution at the final time in $(a)$, and the initial condition for $(c)$ and $(d)$ is the solution at the final time in $(b)$.

on a periodic domain, [-300, 300]. The resulting solution at $t=10000$ is used as an initial condition for the computations with the presence of a turbulent gas. For figure 26(a), $\Theta=1.26$, which corresponds to $U^{*}=1.50 \mathrm{~m} \mathrm{~s}^{-1}$. The amplitude of the pulses increases as time progresses. For figure $26(b), \Theta=1.89$, which corresponds to $U^{*}=1.83 \mathrm{~m} \mathrm{~s}^{-1}$, and the initial condition is the solution obtained at the final time in figure 26(a). We now see that the amplitude of the pulses increases further and the pulses slow down. For figure $26(c), \Theta=2.52$, which corresponds to $U^{*}=2.11 \mathrm{~m} \mathrm{~s}^{-1}$, and this value is close to the flooding gas friction velocity obtained in the previous section by computing periodic travelling waves. The initial condition is the solution obtained at the final time in figure $26(b)$. The amplitude of the pulses increases further 
and the pulses eventually become stationary. This corresponds to the onset of flooding. In contrast, if $\Theta$ is increased from 1.5 to 2.5 , we observe pulses of smaller amplitude than those in figure 26(c) travelling in the negative $x$-direction. This is observed in figure 26(d), where $\Theta=3.15$, which corresponds to $U^{*}=2.36 \mathrm{~m} \mathrm{~s}^{-1}$, while the initial condition is the solution obtained at the final time in figure 26(b). The observed behaviour is in qualitative agreement with, for example, the experimental findings of Semyonov (1944) on gas-liquid flows in a tube, as described in the Introduction.

Of course, as the wave amplitude keeps on increasing the wave will eventually escape the long-wave description. The normal stresses will no longer be a higherorder effect compared with the tangential ones while quite likely a recirculation zone will appear below the wave hump. In this limit, the full equations for the liquid and the gas will have to be used without any approximations.

\section{Discussion}

We have investigated the dynamics of a thin laminar liquid film flowing under the action of gravity down the lower wall of an inclined channel in the presence of a turbulent gas flowing above the film. The solution of the full system of equations describing the gas-liquid flow is an almost formidable problem. However, through a number of assumptions, analytical progress is possible. These assumptions are that the dynamic viscosity of the gas is much smaller than that of the liquid, the typical velocity in the liquid and of the interfacial waves is much smaller than the typical velocity in the gas and the turbulent fluctuations in the gas for the gas-liquid flow decay in the same way as the fluctuations in the gas flowing over a rigid wall. These allow a substantial simplification, namely to consider the gas problem independently to that for the liquid by modelling the interface as a solid wall.

By focusing on the gas problem first, we used a simple mixing-length model and found the perturbations to the pressure and tangential stresses at the interface imposed by the turbulent gas. We worked in curvilinear boundary-layer coordinates first introduced by Benjamin (1959) and followed the approach of Thorsness et al. (1978), who neglected waviness-induced normal Reynolds stresses in their so-called model A. This approach is the simplest one that provides us with sufficiently good agreement with experiments on turbulent flows over wavy walls (see, for example, Thorsness et al. 1978, for the discussion of various approaches) as we demonstrated here by using a coordinate system which approaches a Cartesian one away from the wall and hence is natural for the particular geometry.

We subsequently analysed the problem for the liquid film and derived model equations describing the dynamics of the interface, e.g. boundary-layer equations and a long-wave model. The weakly nonlinear analysis for the latter model resulted in a modified Kuramoto-Sivashinsky equation with an additional term compared with the usual Kuramoto-Sivashinsky equation. This term is due to the presence of the turbulent gas. We found that, for the counter-current gas-liquid flow, the turbulent gas effect is to destabilise the long waves, to stabilise the short ones and to add dispersion. Time-dependent computations for the weakly nonlinear model revealed that the turbulent gas can regularise the dynamics of the usual spatiotemporal chaos of the Kuramoto-Sivashinsky equation in favour of regular trains of travelling pulses of approximately the same shape. The situation is then similar to that observed with the generalised Kuramoto-Sivashinsky equation, the usual Kuramoto-Sivashinsky equation with a third-derivative term (e.g Kawahara 1983; Duprat et al. 2009; Tseluiko et al. 2010a,b). 
Both the weakly nonlinear prototype and the long-wave equation are valid sufficiently close to criticality. To describe nonlinear waves far from criticality, we developed a model based on the IBL approximation for free-falling films. The starting point for this approximation is the first-order boundary-layer equations and a selfsimilar semi-parabolic profile for the fluid velocity. By combining the long-wave approximation and a simple Galerkin projection with just one test function (the semiparabolic profile itself), we obtained a two-equation IBL model for the film thickness and streamwise flow rate which, for the case of free-falling films, provides a good description of the film dynamics sufficiently far from criticality (e.g. Ruyer-Quil \& Manneville 2000). It is then used in our problem for a systematic investigation of the flooding phenomenon in counter-current gas-liquid flows.

As the gas flow rate is increased, the film starts to travel upwards while just before wave reversal the amplitude of the waves grows rapidly. We confirmed the existence of large-amplitude stationary waves by computing travelling waves for our IBL approximation using a helium-methanol system in an inclined channel as a putative experimental set-up. The travelling waves were computed on periodic domains keeping the volume fixed. We found that as the gas flow velocity is increased, the amplitude of the waves grows and, at the same time, the velocity of the waves decreases and tends to zero at a certain critical gas velocity, which corresponds to the onset of flooding. Also, the critical gas velocity decreases as the liquid Reynolds number is increased, as was observed experimentally (e.g. Semyonov 1944). Our travelling-wave results were confirmed by time-dependent computations of the IBL model.

We acknowledge financial support from the Engineering and Physical Sciences Research Council of England through grant no. EP/F009194.

\section{Appendix. Analysis in orthogonal boundary-layer coordinates}

Here, we briefly discuss curvilinear coordinates. For simplicity, we denote the Cartesian coordinates by $\left(x_{1}, x_{2}\right) \equiv(x, y)$ and curvilinear coordinates by $\left(\xi_{1}, \xi_{2}\right)$. We have

for $i=1,2$, and

$$
x_{i}=x_{i}\left(\xi_{1}, \xi_{2}\right),
$$

$$
\xi_{j}=\xi_{j}\left(x_{1}, x_{2}\right),
$$

for $j=1,2$. Next, the covariant basis is defined by

$$
\boldsymbol{g}_{i}=\frac{\partial \boldsymbol{x}}{\partial \xi_{i}}=\frac{\partial x_{j}}{\partial \xi_{i}} \hat{\boldsymbol{x}}_{j}
$$

where $i, j=1,2$ and the Einstein summation convention is used, i.e. there is a summation over an index that appears twice, i.e. index $j$ in the latter expression. Also, $\hat{\boldsymbol{x}}_{i}$ denotes a unit vector pointing in the direction of $x_{i}, i=1,2$. The metric coefficients (entries of the metric tensor) are defined by

$$
g_{i j}=\boldsymbol{g}_{i} \cdot \boldsymbol{g}_{j}=\frac{\partial x_{k}}{\partial \xi_{i}} \frac{\partial x_{k}}{\partial \xi_{j}} .
$$

We will assume that $\left(\xi_{1}, \xi_{2}\right)$ is an orthogonal basis, i.e. the metric tensor is diagonal. The normalised covariant basis is

$$
\hat{\boldsymbol{\xi}}_{i}=\frac{1}{h_{i}} \boldsymbol{g}_{i},
$$

for $i=1,2$, where $h_{i}=\sqrt{g_{i i}}$ are the scale factors, also called Lamé coefficients. 
The transformation from the 'old' orthonormal basis $\left(\hat{\boldsymbol{x}}_{1}, \hat{\boldsymbol{x}}_{2}\right)$ to the 'new' orthonormal basis $\left(\hat{\boldsymbol{\xi}}_{1}, \hat{\boldsymbol{\xi}}_{2}\right)$ is given by

$$
\hat{\boldsymbol{\xi}}_{j}=\gamma_{i j} \hat{\boldsymbol{x}}_{i}
$$

where

$$
\gamma_{i j}=\frac{1}{h_{j}} \frac{\partial x_{i}}{\partial \xi_{j}}
$$

(here, no summation is assumed with respect to $j$ ). Note that the matrix $\boldsymbol{\Gamma}=\left(\gamma_{i j}\right)$ is an orthogonal one, i.e. $\boldsymbol{\Gamma} \boldsymbol{\Gamma}^{\mathrm{T}}=\boldsymbol{\Gamma}^{\mathrm{T}} \boldsymbol{\Gamma}=\boldsymbol{I}$ or, equivalently, $\gamma_{i k} \gamma_{j k}=\gamma_{k i} \gamma_{k j}=\delta_{i j}$. We will additionally assume that $\operatorname{det}(\boldsymbol{\Gamma})=1$, i.e. $\boldsymbol{\Gamma}$ is a special orthogonal matrix, which implies $\gamma_{11}=\gamma_{22}$ and $\gamma_{21}=-\gamma_{12}$. Next, let us indicate how the components of a vector and a tensor field of rank 2 are transformed. Let $\boldsymbol{u}=u_{i} \hat{\boldsymbol{x}}_{i}=\bar{u}_{i} \hat{\boldsymbol{\xi}}_{i}$ and $\boldsymbol{\tau}=\tau_{i j} \hat{\boldsymbol{x}}_{i} \hat{\boldsymbol{x}}_{j}=\bar{\tau}_{i j} \hat{\boldsymbol{\xi}}_{i} \hat{\boldsymbol{\xi}}_{j}$. Then,

$$
u_{i}=\gamma_{i j} \bar{u}_{j}, \quad \bar{u}_{j}=\gamma_{i j} u_{i},
$$

and

$$
\tau_{i j}=\gamma_{i k} \gamma_{j l} \bar{\tau}_{k l}, \quad \bar{\tau}_{k l}=\gamma_{i k} \gamma_{j l} \tau_{i j}
$$

Finally, we discuss an equation satisfied by the streamfunction for the incompressible Reynolds-averaged Navier-Stokes equations. Let $\psi$ be the streamfunction, i.e.

$$
\boldsymbol{U}=\psi_{x_{2}} \hat{\boldsymbol{x}}_{1}-\psi_{x_{1}} \hat{\boldsymbol{x}}_{2}=\frac{1}{h_{2}} \psi_{\xi_{2}} \hat{\boldsymbol{\xi}}_{1}-\frac{1}{h_{1}} \psi_{\xi_{1}} \hat{\boldsymbol{\xi}}_{2},
$$

or, equivalently, the components of the velocity field have the following form:

$$
U_{1}=\psi_{x_{2}}, \quad U_{2}=-\psi_{x_{1}}
$$

in Cartesian coordinates, and

$$
\bar{U}_{1}=\frac{1}{h_{2}} \psi_{\xi_{2}}, \quad \bar{U}_{2}=-\frac{1}{h_{1}} \psi_{\xi_{1}},
$$

in curvilinear coordinates. Then, on eliminating the pressure from the incompressible Reynolds-averaged Navier-Stokes equations, the following equation for the streamfunction is obtained:

$$
\nabla^{4} \psi=-\frac{1}{h_{1} h_{2}} \frac{\partial\left(\psi, \nabla^{2} \psi\right)}{\partial\left(\xi_{1}, \xi_{2}\right)}-\mathscr{R},
$$

where

$$
\nabla^{2}=\frac{1}{h_{1} h_{2}}\left[\frac{\partial}{\partial \xi_{1}}\left(\frac{h_{2}}{h_{1}} \frac{\partial}{\partial \xi_{1}}\right)+\frac{\partial}{\partial \xi_{2}}\left(\frac{h_{1}}{h_{2}} \frac{\partial}{\partial \xi_{2}}\right)\right], \quad \nabla^{4}=\left(\nabla^{2}\right)^{2}
$$

and

$$
\frac{\partial\left(f_{1}, f_{2}\right)}{\partial\left(\xi_{1}, \xi_{2}\right)}=\frac{\partial f_{1}}{\partial \xi_{1}} \frac{\partial f_{2}}{\partial \xi_{2}}-\frac{\partial f_{1}}{\partial \xi_{2}} \frac{\partial f_{2}}{\partial \xi_{1}} .
$$

Here $\mathscr{R}$ is the contribution due to Reynolds stresses, which in Cartesian coordinates has the form

$$
\mathscr{R}=\tau_{11 x_{1} x_{2}}+\tau_{12 x_{2} x_{2}}-\tau_{12 x_{1} x_{1}}-\tau_{22 x_{1} x_{2}} .
$$


To obtain $\mathscr{R}$ in curvilinear coordinates, we can use the transformation law $\tau_{i j}=\gamma_{i k} \gamma_{j l} \bar{\tau}_{k l}$ and the fact that

$$
\begin{aligned}
\left(\begin{array}{l}
\partial / \partial x_{1} \\
\partial / \partial x_{2}
\end{array}\right) & =\left(\begin{array}{ll}
\partial \xi_{1} / \partial x_{1} & \partial \xi_{2} / \partial x_{1} \\
\partial \xi_{1} / \partial x_{2} & \partial \xi_{2} / \partial x_{2}
\end{array}\right)\left(\begin{array}{l}
\partial / \partial \xi_{1} \\
\partial / \partial \xi_{2}
\end{array}\right)=\left(\begin{array}{ll}
\partial x_{1} / \partial \xi_{1} & \partial x_{2} / \partial \xi_{1} \\
\partial x_{1} / \partial \xi_{2} & \partial x_{2} / \partial \xi_{2}
\end{array}\right)^{-1}\left(\begin{array}{l}
\partial / \partial \xi_{1} \\
\partial / \partial \xi_{2}
\end{array}\right) \\
& =\left(\begin{array}{cc}
\gamma_{22} / h_{1} & -\gamma_{21} / h_{2} \\
-\gamma_{12} / h_{1} & \gamma_{11} / h_{2}
\end{array}\right)\left(\begin{array}{l}
\partial / \partial \xi_{1} \\
\partial / \partial \xi_{2}
\end{array}\right)=\left(\begin{array}{ll}
\gamma_{11} / h_{1} & \gamma_{12} / h_{2} \\
\gamma_{21} / h_{1} & \gamma_{22} / h_{2}
\end{array}\right)\left(\begin{array}{l}
\partial / \partial \xi_{1} \\
\partial / \partial \xi_{2}
\end{array}\right),
\end{aligned}
$$

where matrix notations have been used, i.e.

$$
\frac{\partial}{\partial x_{i}}=\frac{\gamma_{i j}}{h_{j}} \frac{\partial}{\partial \xi_{j}}
$$

Therefore,

$$
\begin{aligned}
\mathscr{R}=\frac{\gamma_{1 i}}{h_{i}} \frac{\partial}{\partial \xi_{i}} & \left(\frac{\gamma_{2 j}}{h_{j}} \frac{\partial}{\partial \xi_{j}}\left(\gamma_{1 k} \gamma_{1 l} \bar{\tau}_{k l}\right)\right)+\frac{\gamma_{2 i}}{h_{i}} \frac{\partial}{\partial \xi_{i}}\left(\frac{\gamma_{2 j}}{h_{j}} \frac{\partial}{\partial \xi_{j}}\left(\gamma_{1 k} \gamma_{2 l} \bar{\tau}_{k l}\right)\right) \\
& -\frac{\gamma_{1 i}}{h_{i}} \frac{\partial}{\partial \xi_{i}}\left(\frac{\gamma_{1 j}}{h_{j}} \frac{\partial}{\partial \xi_{j}}\left(\gamma_{1 k} \gamma_{2 l} \bar{\tau}_{k l}\right)\right)-\frac{\gamma_{1 i}}{h_{i}} \frac{\partial}{\partial \xi_{i}}\left(\frac{\gamma_{2 j}}{h_{j}} \frac{\partial}{\partial \xi_{j}}\left(\gamma_{2 k} \gamma_{2 l} \bar{\tau}_{k l}\right)\right) .
\end{aligned}
$$

\section{REFERENCES}

Benjamin, T. B. 1957 Wave formation in laminar flow down an inclined plane. J. Fluid Mech. 2, $554-574$.

Benjamin, T. B. 1959 Shearing flow over a wavy boundary. J. Fluid Mech. 6, 161-205.

Chang, H.-C. \& Demekhin, E. A. 2002 Complex Wave Dynamics on Thin Films. Elsevier Scientific.

Chang, H.-C., Demekhin, E. A. \& Kopelevich, D. I. 1993 Nonlinear evolution of waves on a vertically falling film. J. Fluid Mech. 250, 433-480.

Craster, R. V. \& Matar, O. K. 2009 Dynamics and stability of thin liquid films. Rev. Mod. Phys. 81, 1131-1198.

Demekhin, E. A. 1981 Nonlinear waves in a liquid film entrained by a turbulent gas stream. Fluid Dyn. 16, 188-193.

Dietze, G. F., Al-Sibai, F. \& Kneer, R. 2009 Experimental study of flow separation in laminar falling liquid films. J. Fluid Mech. 637, 73-104.

Dietze, G. F., LeefKen, A. \& KNeER, R. 2008 Investigation of the backflow phenomenon in falling liquid films. J. Fluid Mech. 595, 435-459.

Drosos, E. I. P., Paras, S. V. \& Karabelas, A. J. 2006 Counter-current gas-liquid flow in a vertical narrow channel - Liquid film characteristics and flooding phenomena. Intl J. Multiphase Flow 32, 51-81.

Dukler, A. E. \& SMith, L. 1979 Two-phase interactions in countercurrent flow: studies of the flooding mechanism. Аnпи. Rep. NUREG/CR-0617. U.S. Nuclear Regulatory Commission, Washington, DC.

Duprat, C., Giorgiutti-Dauphiné, F., Tseluiko, D., Saprykin, S. \& Kalliadasis, S. 2009 Liquid film coating a fiber as a model system for the formation of bound states in active dispersivedissipative nonlinear media. Phys. Rev. Lett. 103, 234501.

Guguchinin, V. V., Demekhin, E. A., Kalugin, G. N., Markovich, É. É. \& Pikin, V. G. 1979 Linear and nonlinear stability of combined plane-parallel flow of a film of liquid and gas. Fluid Dyn. 14, 26-31.

Homsy, G. M. 1974 Model equations for wavy viscous film flow. In Lectures in Applied Mathematics: Nonlinear Wave Motion (ed. A. Newell), vol. 15, pp. 191-194. AMS Providence.

Jayanti, S., Tokarz, A. \& Hewitt, G. F. 1996 Theoretical investigation of the diameter effect on flooding in countercurrent flow. Intl J. Multiphase Flow 22, 307-324. 
Jurman, L. A. \& McCready, M. J. 1989 Study of waves on thin liquid films sheared by turbulent gas flows. Phys. Fluids A 1, 522-536.

Kalliadasis, S., Demekhin, E. A., Ruyer-Quil, C. \& Velarde, M. G. $2003 a$ Thermocapillary instability and wave formation on a film falling down a uniformly heated plane. J. Fluid Mech. 492, 303-338.

Kalliadasis, S., KiYashko, A. \& Demeknin, E. A. $2003 b$ Marangoni instability of a thin liquid film heated from below by a local heat source. J. Fluid Mech. 475, 377-408.

Kalliadasis, S. \& Thiele, U. (Ed.) 2007 Thin Films of Soft Matter. Springer.

KaPITZA, P. L. 1948 Wave flow of thin layers of a viscous fluid: I. Free flow; II. Fluid flow in the presence of continuous gas flow and heat transfer. In Collected Papers of P. L. Kapitza (1965) (ed. D. Ter Haar), pp. 662-689. Pergamon (Oxford).

KaWAHARA, T. 1983 Formation of saturated solitons in a nonlinear dispersive system with instability and dissipation. Phys. Rev. Lett. 51, 381-383.

Larson, T. K., Оh, C. H. \& Chapman, J. C. 1994 Flooding in a thin rectangular slit geometry representative of ATR fuel assembly side-plate flow channels. Nucl. Engng Des. 152, 277-285.

LeE, S. C. \& BANKofF, S. G. 1984 Parametric effects on the onset of flooding in flat-plate geometries. Intl J. Heat Mass Transfer 27, 1691-1700.

LiN, S. P. 1974 Finite amplitude side-band stability of a viscous film. J. Fluid Mech. 63, 417-429.

Malamataris, N. A. \& Balakotaiah, V. 2008 Flow structure underneath the large amplitude waves of a vertically falling film. AIChE J. 54, 1725-1740.

Maron, D. M. \& Dukler, A. E. 1984 Flooding and upward film flow in vertical tubes. II. Speculations on film flow mechanisms. Intl J. Multiphase Flow 10, 599-621.

McQuillan, K. W., Whalley, P. B. \& Hewitt, G. F. 1985 Flooding in veritcal two-phase flow. Intl J. Multiphase Flow 11, 741-760.

Meza, C. E. \& Balakotaiah, V. 2008 Modeling and experimental studies of large amplitude waves on vertically falling films. Chem. Engng Sci. 63, 4704-4734.

Miles, J. W. 1957 On generation of surface waves by shear flows. J. Fluid Mech. 3, 185-204.

Mouza, A. A., Pantzali, M. N. \& Paras, S. V. 2005 Falling film and flooding phenomena in small diameter vertical tubes: the influence of liquid properties. Chem. Engng Sci. 60, 4981-4991.

Nepomnyashchy, A. A. 1974 Stability of wave regimes in a film flowing down an inclined plane. Fluid Dyn. 9, 354-359.

Oron, A., Davis, S. H. \& Bankoff, S. G. 1997 Long-scale evolution of thin liquid films. Rev. Mod. Phys. 69, 931-980.

Oron, A. \& Gottlieb, O. 2002 Nonlinear dynamics of temporally excited falling liquid films. Phys. Fluids 14, 2622-2636.

Pantzali, M. N., Mouza, A. A. \& Paras, S. V. 2008 Counter-current gas-liquid flow and incipient flooding in inclined small diameter tubes. Chem. Engng Sci. 63, 3966-3978.

Peng, C.-A., Jurman, L. A. \& McCready, M. J. 1991 Formation of solitary waves on gas-sheared liquid layers. Intl J. Multiphase Flow 17, 767-782.

Pereira, A. \& Kalliadasis, S. 2008 Dynamics of a falling film with solutal Marangoni effect. Phys. Rev. E 78, 036312.

Pumir, A., Manneville, P. \& Pomeau, Y. 1983 On solitary waves running down an inclined plane. J. Fluid Mech. 135, 27-50.

Ruyer-Quil, C. \& Manneville, P. 1998 Modeling film flows down inclined planes. Eur. Phys. J. B 6, 277-292.

Ruyer-Quil, C. \& Manneville, P. 2000 Improved modeling of flows down inclined planes. Eur. Phys. J. B 15, 357-369.

Ruyer-Quil, C. \& Manneville, P. 2002 Further accuracy and convergence results on the modeling of flows down inclined planes by weighted-residual approximations. Phys. Fluids 14, 170-183.

Ruyer-Quil, C., Scheid, B., Kalliadasis, S., Velarde, M. G. \& Zeytounian, R. Kh. 2005 Thermocapillary long waves in a liquid film flow. Part 1. Low-dimensional formulation. J. Fluid Mech. 538, 199-222.

Ruyer-Quil, C., Treveleyan, P., Giorgiutti-Dauphiné, F., Duprat, C. \& Kalliadasis, S. 2008 Modelling film flows down a fiber. J. Fluid Mech. 603, 431-462.

Scheid, B., Ruyer-Quil, C., Kalliadasis, S., Velarde, M. G. \& Zeytounian, R. Kh. 2005 Thermocapillary long waves in a liquid film flow. Part 2. Linear stability and nonlinear waves. J. Fluid Mech. 538, 233-244. 
Scheid, B., Ruyer-Quil, C., Thiele, U., Kabov, O. A., Legros, J. C. \& Colinet, P. 2004 Validity domain of the Benney equation including Marangoni effect for closed and open flows. J. Fluid Mech. 527, 303-335.

Schlichting, H. 2000 Boundary-Layer Theory. Springer.

Semyonov, P. A. 1944 Flows of thin liquid films. J. Tech. Phys. (in Russian) 14, 427-437.

Shearer, C. J. \& Davidson, J. F. 1965 The investigation of a standing wave due to gas blowing upwards over a liquid film; its relation to flooding in wetted-wall columns. J. Fluid Mech. 22, 321-335.

ShKadov, V. Ya. 1967 Wave flow regimes of thin layer of viscous fluid subject to gravity. Fluid Dyn. 2, 29-34.

STAINTHORP, F. P. 1967 The effect of co-current and counter-current air flow on the wave properties of falling liquid films. Trans. Inst. Chem. Engrs 45, 372-382.

Sudo, Y. 1996 Mechanisms and effects of predominant parameters regarding limitation of falling water in vertical counter-current two-phase flow. J. Heat Transfer 118, 715-724.

Thorsness, C. B., Morrisroe, P. E. \& Hanratty, T. J. 1978 A comparison of linear theory with measurements of the variation of shear stress along a solid wave. Chem. Engng Sci. 33, 579-592.

Trevelyan, P. M. J. \& Kalliadasis, S. 2004a Dynamics of a reactive falling film at large Péclet numbers. II. Nonlinear waves far from criticality: Integral-boundary-layer approximation. Phys. Fluids 16, 3209-3226.

Trevelyan, P. M. J. \& Kalliadasis, S. $2004 b$ Wave dynamics on a thin-liquid film falling down a heated wall. J. Engng Maths 50, 177-208.

Trevelyan, P. M. J., Scheid, B., Ruyer-Quil, C. \& Kalliadasis, S. 2007 Heated falling films. J. Fluid Mech. 592, 295-334.

Trifonov, YU. YA. 2010 Counter-current gas-liquid wavy film flow between the vertical plates analyzed using the Navier-Stokes equations. AIChE J. 56, 1975-1987.

Tseluiko, D., Saprykin, S., Duprat, C., Giorgiutti-Dauphiné, F. \& Kalliadasis, S. $2010 a$ Pulse dynamics in low-Reynolds-number interfacial hydrodynamics: Experiments and theory. Physica D 239, 2000-2010.

Tseluiko, D., Saprykin, S. \& Kalliadasis, S. $2010 b$ Interaction of solitary pulses in active dispersive-dissipative media. Proc. Est. Acad. Sci. 59, 139-144.

Yıн, C.-H. 1963 Stability of liquid flow down an inclined plane. Phys. Fluids 6, 321-334.

ZAPKE, A. \& KRÖGER, D. G. $2000 a$ Countercurrent gas-liquid flow in inclined and vertical ducts. I. Flow patterns, pressure drop characteristics and flooding. Intl J. Multiphase Flow 26, $1439-1455$.

ZAPKe, A. \& KRöGer, D. G. $2000 b$ Countercurrent gas-liquid flow in inclined and vertical ducts. II. The validity of the Froude-Ohnesorge number correlation for flooding. Intl J. Multiphase Flow 26, 1457-1468.

Zilker, D. P., Cook, G. W. \& Hanratty, T. J. 1977 Influence of the amplitude of a solid wavy wall on a turbulent flow. Part 1. Non-separated flows. J. Fluid Mech. 82, 29-51. 